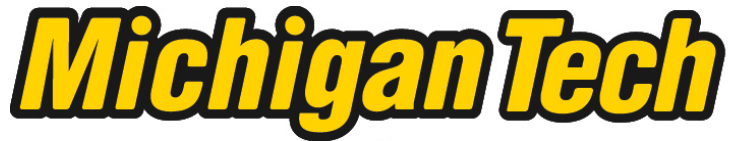 \\ Michigan Technological University Create the Future Digital Commons @ Michigan Tech
}

Computational simulations of latent heat thermal energy storage systems - with innovative and first-principles based simulation for the underlying unsteady melting (and solidification) processes

Rohan Achyut Gumaste

Michigan Technological University

Follow this and additional works at: https://digitalcommons.mtu.edu/etds

Part of the Mechanical Engineering Commons

Copyright 2011 Rohan Achyut Gumaste

Recommended Citation

Gumaste, Rohan Achyut, "Computational simulations of latent heat thermal energy storage systems - with innovative and first-principles based simulation for the underlying unsteady melting (and solidification) processes", Master's Thesis, Michigan Technological University, 2011.

https://doi.org/10.37099/mtu.dc.etds/370

Follow this and additional works at: https://digitalcommons.mtu.edu/etds

Part of the Mechanical Engineering Commons 


\title{
COMPUTATIONAL SIMULATIONS OF LATENT HEAT THERMAL ENERGY \\ STORAGE SYSTEMS - WITH INNOVATIVE AND FIRST-PRINCIPLES BASED SIMULATION FOR THE UNDERLYING UNSTEADY MELTING (AND SOLIDIFICATION) PROCESSES
}

\author{
by \\ Rohan Achyut Gumaste \\ A THESIS \\ Submitted in partial fulfillment of the requirements for the degree of \\ MASTER OF SCIENCE \\ (Mechanical Engineering)
}

MICHIGAN TECHNOLOGICAL UNIVERSITY

2011

(C) 2011 Rohan Achyut Gumaste 
This thesis, "Computational Simulations of Latent Heat Thermal Energy Storage Systems - with Innovative and First-principles based Simulation for the Underlying Unsteady Melting (and Solidification) Processes," is hereby approved in partial fulfillment of the requirements for the Degree of MASTER OF SCIENCE IN MECHANICAL ENGINEERING

Department Of Mechanical-Engineering Mechanics

Signatures:

Thesis Advisor Dr. Amitabh Narain

Committee Member Dr. Charles Margraves

Committee Member Dr. Robert Kolkka

Department Chair Dr. William W. Predebon

Date 


\section{Table of Contents}

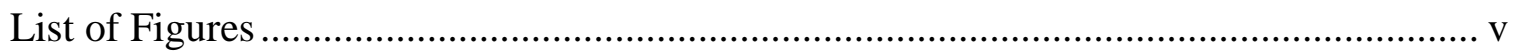

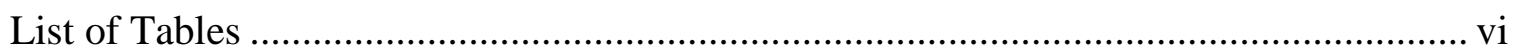

List of Symbols ................................................................................................... vii

List of Abbreviations .............................................................................................. vii

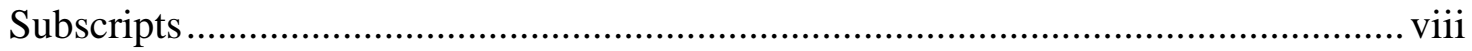

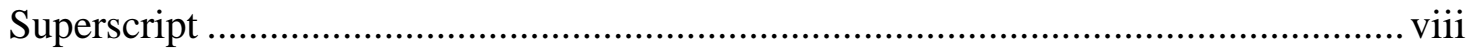

Abstract ............................................................................................................ ix

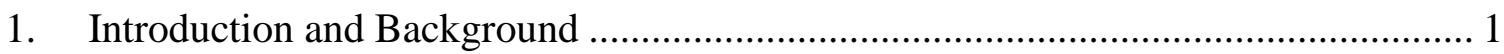

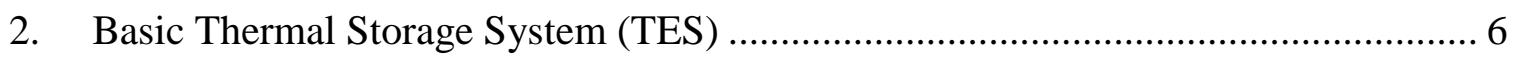

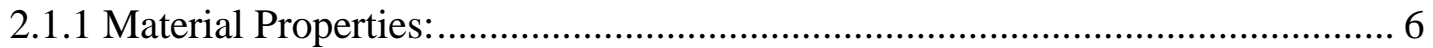

2.1.2 Dimensions and Selected boundary conditions ......................................... 8

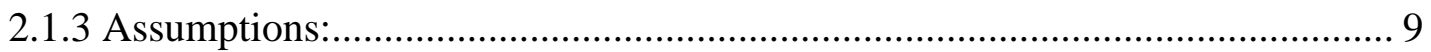

2.2 Description of the Basic Thermal Storage System (TES) ………........................... 9

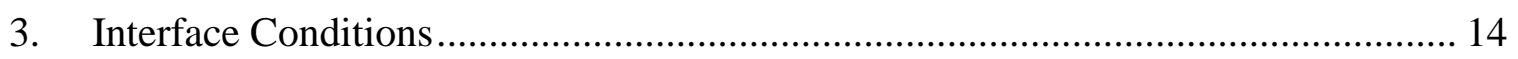

3.1 Physics at the Melt-Solid Interface ............................................................. 14

3.2 Physics at the HTF - Pipe - PCM interface ................................................. 15

4. Conjugate Heat Transfer Problems for the LHTES System ..................................... 16

4.1 Nature of Conjugate Heat Transfer Problems and their respective Domains ......... 16

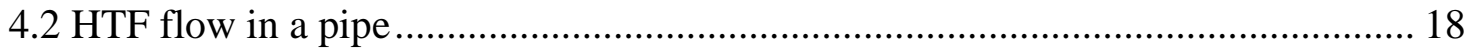

4.3 Other Conjugate Heat Transfer Problems for "Before Melting" Duration............. 20

4.3.1 Unsteady Conduction for the Solid PCM .................................................... 20

4.4 Other Conjugate Heat Transfer Problems for the "Melting" Duration.................... 21

4.4.1 Unsteady Conduction Problem for the Molten PCM....................................... 22

4.4.2 Unsteady Conduction Problem for the Solid PCM on the Other Side of the Melt-front ................................................................................................. 23

5. The Solution Approach for the Conjugate Problems ................................................ 25

5.1 Numerical Solution Approach for the "Before Melting" Duration Conjugate

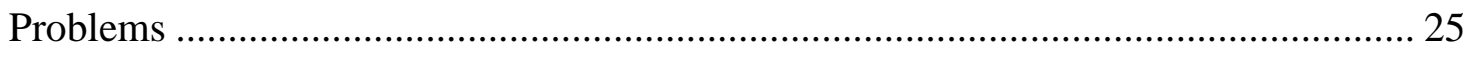

5.2 Numerical Solution Approach for the "Melting" Duration Conjugate Problems ... 26 
6. Convergence and Reliability of the Computed Solution ...................................... 29

6.1 Grid Independence of the Unsteady Solution ........................................... 29

6.2 Independence with respect to the Computational Method used for solving the

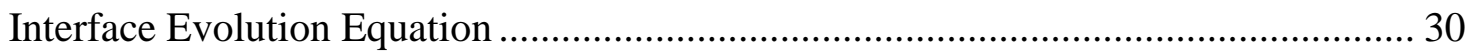

6.3 Results for the "Before Melting" Duration .................................................. 31

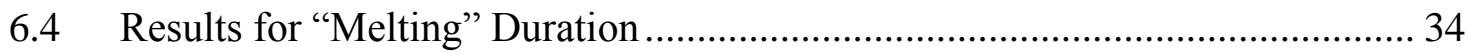

6.5 Results for "Melting" Duration (Natural Convection within the Molten PCM

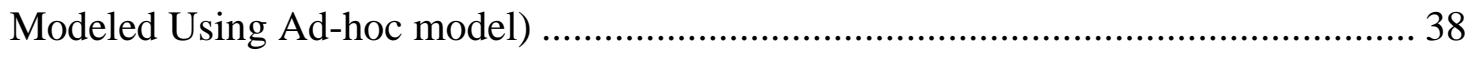

6.6 The Comparison with Analytical Solution for Two Phase Stefan Problem:...... 41

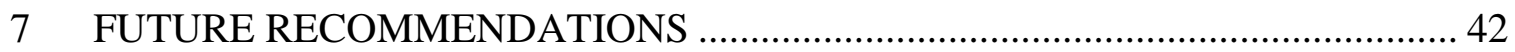

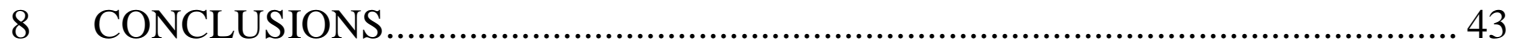

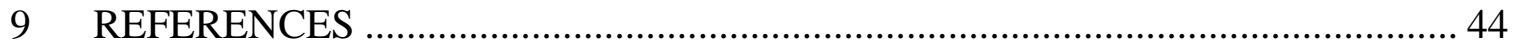

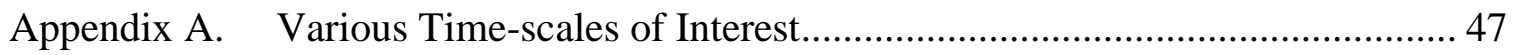

Appendix B. Rapid Encapsulation of the HTF pipe by PCM Melt ............................ 52

Appendix C. Equations Modeling the Physics - based Requirements that must be met

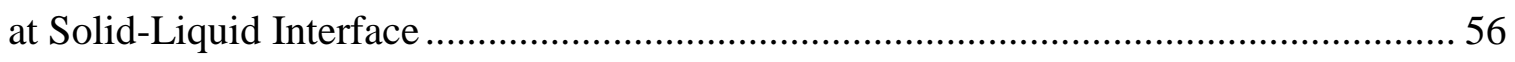

Appendix D. Methods for Solving the Melt-Solid Interface Evolution Equation ........ 61 


\section{List of Figures}

Figure 1.1: Rankine Cycle with Thermal Storage (TES) Unit............................................ 2

Figure 2.1: Geometry for Conjugate Heat Transfer Problem ............................................. 8

Figure 2.2: Thermal Energy Storage (TES) System ..................................................... 10

Figure 2.3: Schematic of Wall Temperature $T w x, t$ versus downstream distance $x$...... 11

Figure 2.4: Schematic Evolution of Interface $\Delta x, t$ versus Downstream Distance x....... 12

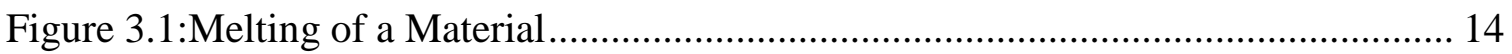

Figure 3.2: Conjugate Heat Transfer Problems for the LHTES System........................... 15

Figure 4.1: Domains for Conjugate Heat Transfer Problem........................................... 17

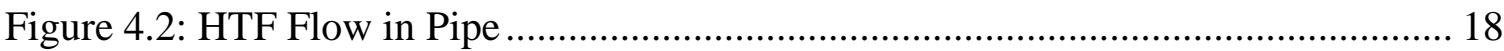

Figure 4.3: Initial and Boundary Conditions for Solid PCM domain................................ 21

Figure 4.4: Initial and Boundary Conditions for Molten PCM domain........................... 22

Figure 4.5: Initial and boundary conditions for solid PCM domain ................................ 24

Figure 6.1: Schematic Representation of Spatial Grids Used ............................................ 29

Figure 6.2: Grid Independence for Predicted Location of Interface $\Delta x, t \ldots \ldots \ldots \ldots \ldots \ldots \ldots \ldots . . . .30$

Figure 6.3: Comparison for Location of Interface $\Delta x, t$ at $t=1 e 4 \ldots \ldots \ldots \ldots \ldots \ldots \ldots \ldots \ldots \ldots . . . .30$

Figure 6.4: Initial Conditions (wall temperature and mean HTF temperature $t=0 \mathrm{~s}$ ) ...... 31

Figure 6.5: Plot Temperature and Mean Temperature (at $\mathrm{t}=0 \mathrm{~s}$ and after first time-step) 32

Figure 6.6: Plot of Wall Temperature $T w(x, t)$ after every 50 time-steps ..................... 32

Figure 6.7: Plot of Mean Temperature Tm $(x, t)$ after every 50 time-steps..................... 33

Figure 6.8: Plot of Wall Flux $q "(x, t)$ after every 50 time-steps .................................. 33

Figure 6.9: Plot of Wall Temperature $T w(x, t M)$ and Mean Temperature $T m(x, t M) .34$

Figure 6.10: Plot of Assumed and Corrected Location of Interface $\Delta x, t=0 \ldots \ldots \ldots \ldots \ldots . .34$

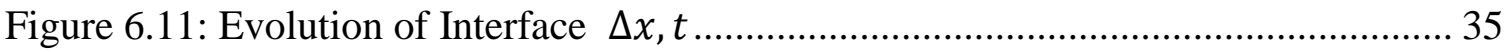

Figure 6.12: Representative plot of the temperature variation in radial direction ............ 36

Figure 6.13: Wall Temperature $T w x, t \mathrm{v} / \mathrm{s}$ downstream distance ("Melting" duration). 36

Figure 6.14: Plots of $\in 1(t)$, against time $t$ for spatial domains of different lengths...... 37

Figure 6.15: Plots of $\in 2(t)$, against time $t$ for spatial domains of different lengths ..... 37

Figure 6.16: Plots of $\eta(t)$, against time $t$ for spatial domains of different lengths ........ 38

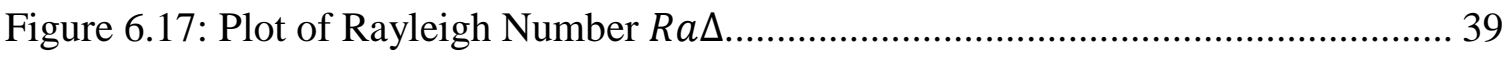

Figure 6.18 : Evolution of Interface $\Delta\left(\mathrm{x}, \mathrm{t}^{\wedge}\right)$ (with natural convection effects) ................ 39

Figure 6.19 : Plots of efficiencies $\varepsilon 1$ for two different cases .......................................... 40

Figure 6.20: Plots of efficiencies $\varepsilon 2$ for two different cases .......................................... 40

Figure 6.21: Plot of Effectiveness for two different cases............................................. 40

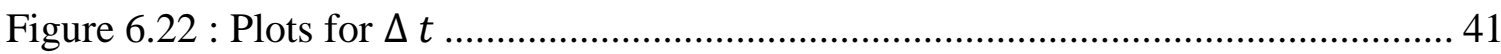

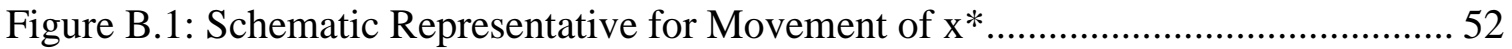

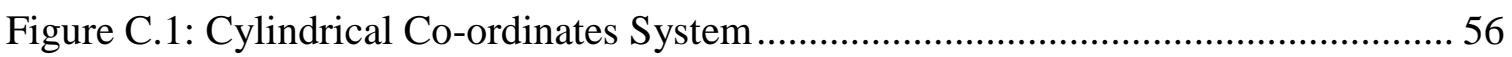




\section{List of Tables}

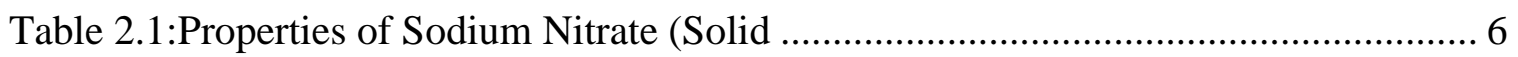

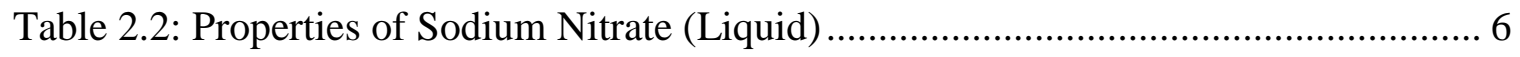

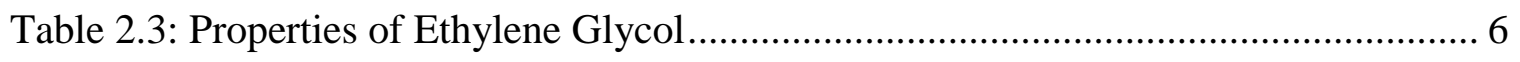

Table 2.4 Selected Dimensions and Boundary Conditions ........................................... 8 


\section{List of Symbols}

$\dot{M}$ : Mass flow rate of heat transfer fluid $(\mathrm{kg} / \mathrm{s})$

$T_{\infty}:$ Far-field temperature (K)

$T_{i n}:$ Inlet temperature of heat transfer fluid $(\mathrm{K})$

$\dot{m}$ : Mass rate of melting of the phase change material $(\mathrm{kg} / \mathrm{s})$

$\Delta$ : Distance of interface between liquid and solid phase from pipe wall

$\mathrm{D}_{1:}$ Diameter of pipe $(\mathrm{m})$

$\mathrm{D}_{2:}$ Diameter of the container (m)

$\mathrm{h}_{\mathrm{fg}}$ : Latent heat of melting $(\mathrm{J} / \mathrm{kg} \mathrm{K})$

L: Length of pipe (m)

r: Distance along radial direction $(\mathrm{m})$

$\mathrm{r}_{1}$ : Radius of a pipe $(\mathrm{m})$

$\mathrm{r}_{2}$ : Radius of a PCM container (m)

T: Temperature $(\mathrm{K})(\mathrm{m})$

V: Velocity $(\mathrm{m} / \mathrm{s})$

$\mathrm{x}$ : Distance along axial direction (m)

$\theta$ : Angle $\left({ }^{\circ}\right)$

$\rho$ : Density $\left(\mathrm{kg} / \mathrm{m}^{3}\right)$ 


\section{List of Abbreviations}

HTF: Heat transfer fluid

PCM: Phase Change Material

TES: Thermal Energy Storage

LHTES: Latent Heat Thermal Energy Storage

Subscripts

1: Molten PCM

2: Solid PCM

F: Heat Transfer Fluid

\section{Superscript}

i: Melt-solid Interface 


\begin{abstract}
This thesis develops an effective modeling and simulation procedure for a specific thermal energy storage system commonly used and recommended for various applications (such as an auxiliary energy storage system for solar heating based Rankine cycle power plant). This thermal energy storage system transfers heat from a hot fluid (termed as heat transfer fluid - HTF) flowing in a tube to the surrounding phase change material (PCM). Through unsteady melting or freezing process, the PCM absorbs or releases thermal energy in the form of latent heat. Both scientific and engineering information is obtained by the proposed first-principle based modeling and simulation procedure. On the scientific side, the approach accurately tracks the moving melt-front (modeled as a sharp liquid-solid interface) and provides all necessary information about the time-varying heat-flow rates, temperature profiles, stored thermal energy, etc. On the engineering side, the proposed approach is unique in its ability to accurately solve - both individually and collectively - all the conjugate unsteady heat transfer problems for each of the components of the thermal storage system. This yields critical system level information on the various time-varying effectiveness and efficiency parameters for the thermal storage system.
\end{abstract}




\section{Introduction and Background}

Increased awareness of air pollution (including accumulation of green houses gases) and energy security issues (arising out of rising prices and future shortage of gasoline, etc) is stimulating research and innovation in energy efficiency and renewable energy sources. Solar radiation as a thermal energy source remains one of the potent renewable energy alternative, considering that solar radiation is freely and abundantly available in many parts of the world. Because of the intermittent nature of this energy supply (absence during night time and limited availability during winter/rainy seasons), new solar power plants must incorporate both the thermal energy storage option (to store unused excess thermal energy for later use when solar energy is not available) as well as other energy utilization (e.g. coal, nuclear, etc) to be effective in economically meeting continuous power generation requirements under varying conditions of the supply and demand.

Among different forms of energy storage (chemical, nuclear, mechanical, thermal, etc), the thermal energy storage systems interest here are called Latent Heat Thermal Energy Storage (LHTES). Though thermal energy can be stored in different forms (such as sensible heat associated with a material's temperature, thermo-chemical energy, latent heat, etc), this study limits itself to LHTES system that employ solid-liquid phase-change processes in suitable phase-change materials (PCMs) that absorb or release latent heat of fusion.

The specific thermal energy storage (TES) considered here is shown in Fig. 1.1 in the context of a specific application involving a solar heating based Rankine cycle power plant. In the specific solar power plant application of Fig. 1.1, the heat from solar energy can either go to the boiler (through path A) for power generation or can be diverted (through path B) to store (as latent heat absorbed during melting of the PCM in the TES) for later use. Later use (not discusses for brevity) is a simple reversal of the phase-change process in which the molten PCM (in the TES of Fig. 1.1) freezes and releases the latent heat which is carried back by reversed flow of the heat transfer fluid (HTF). 


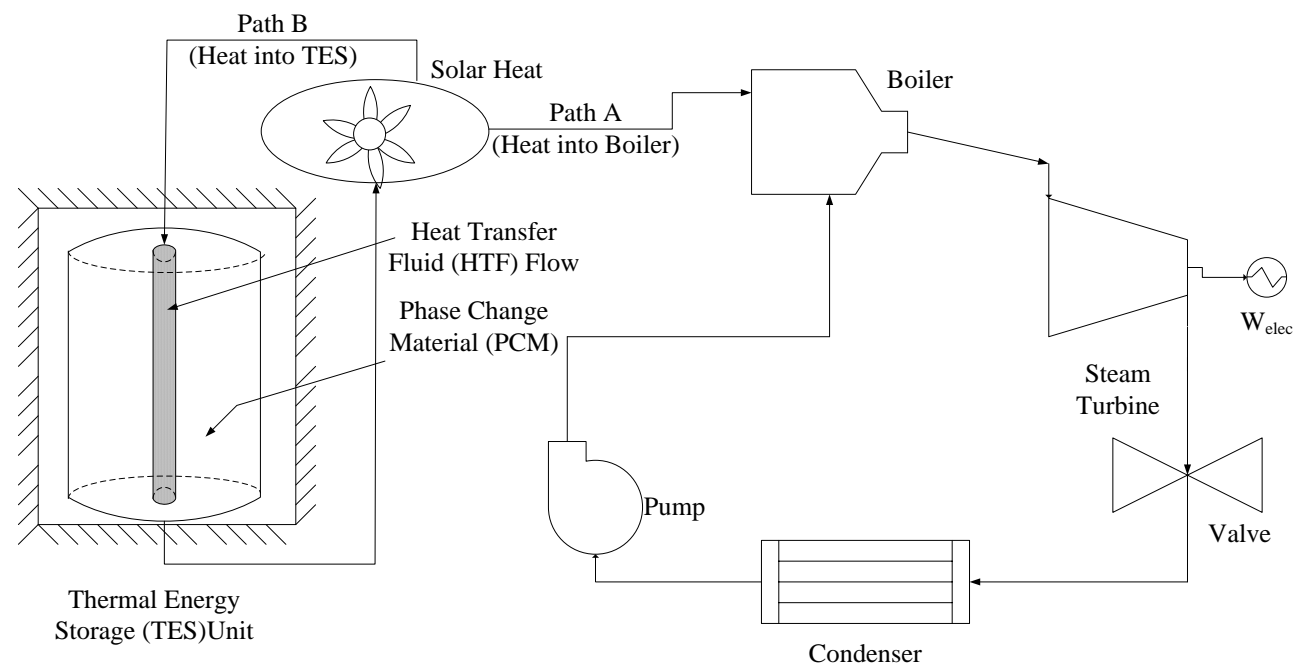

Figure 1.1: Rankine Cycle with Thermal Storage (TES) Unit

Many different types of PCM (organic, inorganic, eutectic, etc see (Zalba 2003; Kenisarin 2007; Sharma 2009; Agyenim 2010) were used in many different thermal energy storage devices to improve the efficiencies of different systems (such as heat pumps, spacecraft thermal management systems, commercial HVAC systems, shipping and packaging, etc). In the context of TES unit in the solar power application of Fig. 1.1, the choice of the PCM is based on careful consideration of its melting temperature, its stability during several melting - freezing cycles, its thermal properties (conductivity, latent heat of fusion, etc), cost, etc. Careful considerations of such issues have been extensively discussed in (Sharma 2009; Agyenim 2010). However, in the context of a TES system application in Fig. 1.1, it is assumed that a suitable choice of PCM exists (such as often used $\mathrm{NaNO}_{3}$ or $\mathrm{KNO}_{3}$ ) and that its properties are known.

Despite the fact that suitable PCM choices exists for energy storage (TES) system of the type used in Fig. 1.1, their low thermal conductivity (often less than $1 \frac{\mathrm{W}}{\mathrm{m}-\mathrm{K}}$ ) create serious limitation on the time-varying effectiveness and efficiency of the TES. Active ongoing research suggests that the effective thermal conductivity of the PCM and performance of the TES can be improved by using one or more of the following:

(i) Use of suitable nano-particles in the PCM matrix to enhance its thermal properties (see (Shin 2009; Shin 2010)) 
(ii) Use of thermo-chemical reactions to supplement the latent heat absorption / release processes in the PCM.

(iii) Use of high thermal conductivity fins embedded in the PCM and the exterior side of tube carrying the HTF (see (Castell 2008; Wang 2010))

(iv) Use of small diameter heat pipes (replacing the fins with even higher effective thermal conductivity) mounted on the exterior of the tube carrying the HTF (see (Robak 2011; Weng 2011)).

Though the basic modeling approach of the this study, the properties of the pure PCM are assumed, these thermal properties (conductivity, heat capacity, etc) can be changed with the help of ad hoc models - to assess the effectiveness and efficiency advantages resulting from employing any of the above approaches for enhancing the performance of the PCM in the TES under consideration.

With regard to modeling and simulation of fundamental and system level melting/ freezing problems at the heart of the analysis for a LHTES of interest, a lot has been done and is known. For example, besides the governing equations for each of the two phases, the mathematical modeling of the physics at the liquid-solid interface is known quite accurately (see (Delhay 1974; Abbott 1989; Narain 2004)) provided equilibrium thermodynamic conditions (as opposed to non-equilibrium thermal conditions) and "sharp" interface models (as opposed to detailed "mushy" zone models) are considered adequate. This is the case for the TES problem of interest here; because of system level results are of primary interest. Despite the accurate knowledge that exists with regard to interface conditions, the found solutions - whether analytical (see (Tzai-Fu 2000; C 2007; Chantasiriwan 2009)), or computational (see (T 2000; J 2004; Shmueli 2010; Wang 2010; Onyejekwe 2011; Ye 2011)) solution procedures of the governing PDEs typically limit themselves to the steady thermal boundary conditions for the heat transfer boundary of the PCM. However, for the LHTES system problem of interest, the heat transfer boundary condition at the interface of the HTF pipe and the PCM experiences unsteady boundary conditions that can only be determined by a conjugate analysis of the transient heat transfer within the PCM and forced convection in HTF flow. With regard to such conjugate analysis, the available results in the existing literature limit themselves 
to the approximate integral approaches (see (Esen 1996; Michels 2007)) which are not sufficient for extracting all the useful information (such as time evolving melt-front locations impact on the total heat stored, efficiency, effectiveness, etc) for the LHTES system. This study removes this hurdle and provides a simulation approach that allows one to obtain both the scientific and engineering information on the LHTES system within the framework of the modeling employed here.

With regard to detailed computational simulation, the approach preferred in the literature is to treat the moving interface problem by an enthalpy formulation approach that applies to both the phases of the PCM. In this method, the interface is captured as a finite thickness "mushy" zone over which the enthalpy $h$ (which appears in the differential form of the energy equation: $\left.\frac{\partial h}{\partial t} \cong \operatorname{div}(k \cdot g r a d T)\right)$ rapidly varies between its values for the liquid and the solid phases. In the limit of shrinking thickness of the mushy zone, one recovers and satisfies all the exact interface conditions (see (Abbott 1989; Delhaye 1974; Narain 2004)) employed in our proposed "sharp" interface modeling approach. If this approach of enthalpy formulation is successful, one does not need to explicitly track the interface. Despite this desired limiting behavior of the "mushy" zone is difficult to attain in practice and one experience oscillations in temperature. This is because a good and robust technique by this approach requires both the smallness of the mesh size (for a shrinking "mushy" zone) as well as a proper discretization scheme for this zone.

The proposed "sharp" interface modeling approach is successful, because modeling approach explicitly satisfies the phase-change physics at the interface while accurately tracking its time evolution (through one of these interface conditions). This ability to accurately satisfy all the physics at the interface (such as mass balance, energy balance, etc) along with the satisfaction of the physics for all components of the system makes our modeling results quite reliable. The interface tracking approach (see (Mitra 2011)) used here for freezing/melting problem sees for the solid PCM as a stationary phase. This very same interface tracking approach has proved to be effective for more complex annular condensing (Mitra 2011) and annular boiling problem - where both the phases move and affect the location of interface. Because of the advantage of the problem simulation 
approach and the ability to implement the solution procedure by a combination of commercially available single-phase simulation tool (COMSOL is used here) and relatively simpler user written codes (a MATLAB) for locating the moving solid-liquid interface, the proposed approach can be relatively easily extended to account for natural convection in the molten phase and finite thermal resistance for the tube wall.

The modeling and simulation tool described here accomplished the following:

- It captures the physics of each of the conjugate heat transfer problem and concurrently yields time-varying solutions for the HTF flow and the melting in the PCM. This yields the requisite information on heat transfer rates, temperature profiles, amount of thermal energy stored in the PCM.

- It tracks the time-varying locations of the interface in the manner that is free from any computational noise in the absence of any physical noise.

- Solutions from this approach satisfy convergence criterion in all the domains (HTF, molten PCM and solid PCM).

- The solutions satisfy all the heat balance, mass balance, thermo-dynamics conditions, etc at each point on the interface.

- It is verified that the solutions are correct in the sense that overall energy balance (first law of thermodynamics) is satisfied for the entire system or any control volume of interest.

- The solutions obtained exhibit the required grid independence behavior with regard to discretization in space and time.

- The methodology is efficient as it does not consume extensive computational time to model both the "before melting" and "melting" duration of interest. 


\section{Basic Thermal Storage System (TES)}

\subsubsection{Material Properties:}

Table 2.1 - 2.2 show properties of solid and liquid phase of Sodium Nitrate respectively. These properties are taken at $306^{\circ} \mathrm{C}$. Solid or liquid sodium Nitrate is assumed to be homogenous and its properties are listed below modeled to be temperature-independent over the range of temperature considered here.

Table 2.1:

Properties of Sodium Nitrate (Solid

\begin{tabular}{|l|l|}
\hline Melting Temperature $\left({ }^{\circ} \mathrm{C}\right)$ & 306 \\
\hline Heat of fusion (melting) $(\mathrm{kJ} / \mathrm{kg})$ & 172 \\
\hline Density $\left(\mathrm{kg} / \mathrm{m}^{3}\right)$ & 2261 \\
\hline Heat Capacity $(\mathrm{kJ} / \mathrm{kgK})$ & 1.10 \\
\hline Thermal Conductivity $(\mathrm{W} / \mathrm{mK})$ & 0.5 \\
\hline
\end{tabular}

(Michels 2007)

Table 2.2:

Properties of Sodium Nitrate (Liquid)

\begin{tabular}{|l|l|}
\hline Density $\left(\mathrm{kg} / \mathrm{m}^{3}\right)$ & 1908 \\
\hline Heat Capacity $(\mathrm{kJ} / \mathrm{kgK})$ & 1.655 \\
\hline Thermal Conductivity $(\mathrm{W} / \mathrm{mK})$ & 0.514 \\
\hline
\end{tabular}

(Bauer 2009); (Peng 2010),

Table 2.3:

Properties of Ethylene Glycol

\begin{tabular}{|l|l|}
\hline Density $\left(\mathrm{kg} / \mathrm{m}^{3}\right)$ & 1113.2 \\
\hline Heat Capacity $(\mathrm{kJ} / \mathrm{kgK})$ & 2.460 \\
\hline Thermal Conductivity (W/mK) & 0.25 \\
\hline Dynamic Viscosity (Pas) & 0.016 \\
\hline Ratio of Specific Heats & 1 \\
\hline
\end{tabular}




\subsubsection{Dimensions and Selected boundary conditions}

Figure 2.1 shows the geometry for the HTF flow in a pipe (of negligible thickness, of diameter $D$ and length $L$ ) in a conjugate heat transfer problem for the annular PCM (inner diameter $D_{1}$ and outer diameter $D_{2}$ ) encapsulating the HTF flow tube. Selected values of representative dimensions and boundary conditions are given in Table 2.4.

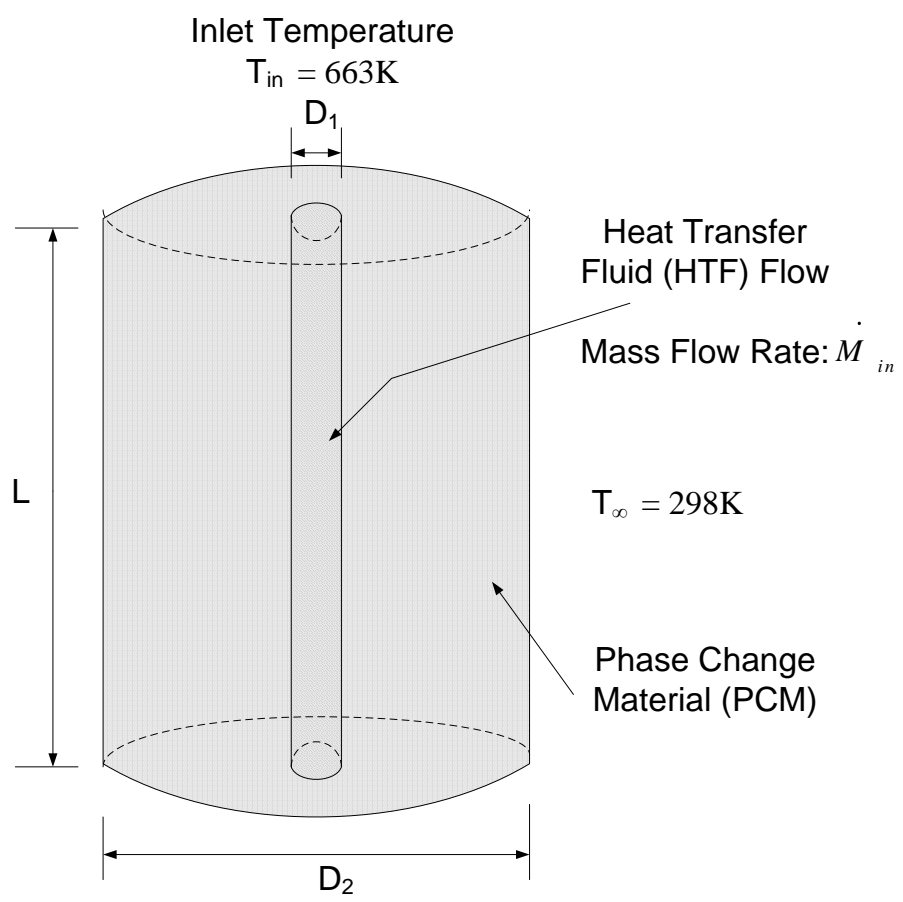

Figure 2.1: Geometry for Conjugate Heat Transfer Problem

Table 2.4

Selected Dimensions and Boundary Conditions

\begin{tabular}{|l|l|}
\hline $\mathrm{D}_{1}(\mathrm{~m})$ & 0.02 \\
\hline $\mathrm{D}_{2}(\mathrm{~m})$ & 0.5 \\
\hline $\mathrm{L}(\mathrm{m})$ & 2 \\
\hline Inlet Temperature of HTF $\left(T_{i n}\right)(\mathrm{K})$ & 663 \\
\hline Inlet Mass flow rate $\left(M_{\text {ln }}\right)\left(\mathrm{kg} / \mathrm{m}^{3}\right)$ & 0.03 (Michels 2007) \\
\hline $\begin{array}{l}\text { The boundary condition for the PCM at outer } \\
\text { diameter } D_{2}\end{array}$ & $\begin{array}{l}\text { Ambient temperature } T_{\infty}=298 K \\
\text { (assumed here) or adiabatic (zero } \\
\text { heat flux) condition }\end{array}$ \\
\hline
\end{tabular}


Solutions for other boundary conditions of this type are possible and only briefly discussed here.

\subsubsection{Assumptions:}

- The problem is axisymmetric (angular variation is absent) and two-dimensional.

- At all times steady flow rate of HTF maintains the inlet temperature $T_{i n}$, which is taken here to be $663 \mathrm{~K}$.

- HTF is an incompressible Newtonian fluid.

- The thermo-physical properties of HTF and PCM are adequately modeled by their representative constant values for the temperature range of interest. For density, specific heat, thermal conductivity, etc the common notations of $\rho, C_{p}, K$, etc are used. The subscripts used for the HTF fluid, molten PCM and the solid PCM are respectively "F", "1", "2". Therefore, $\rho_{F}, \rho_{1}$, and $\rho_{2}$ respectively denote the density of the HTF fluid, the molten PCM, and the solid PCM

- The molten and solid PCM are homogenous and isotropic.

- As pressure in the PCM is approximately constant, phase change occurs at nearly

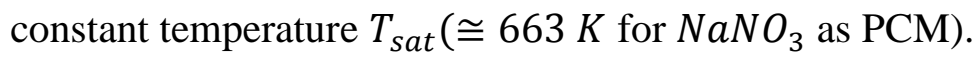

- Natural convection in molten PCM is neglected for this study. It is expected to become important when sufficient amount molten PCM is accumulated. The proposed simulation tool can be enhanced in future to model the natural convection.

\subsection{Description of the Basic Thermal Storage System (TES)}

Figure 2.2 below represents the typical TES problem in systems of interest (such as the one in Fig. 1.1), where the phase change material (PCM) is melted (or solidified) by passage of a hot-fluid (or cold fluid) - termed as heat transfer fluid (HTF) - through a tube imbedded in the PCM. For the system described in Fig. 1.1, the melting occurs during charging cycle in which the HTF cools down as it flows downstream whereas the PCM heats up. Modeling and prediction of this charging cycle is the subject of interest here. Discharging/freezing cycle is not considered here. However, discharging cycle's 


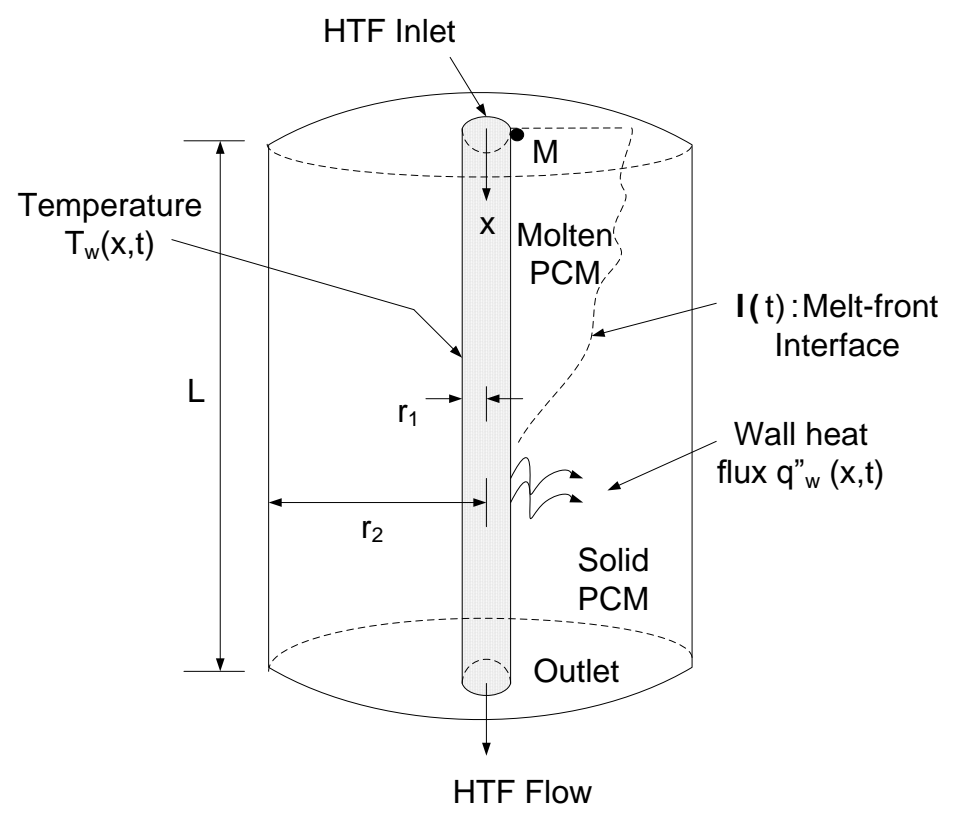

Figure 2.2: Thermal Energy Storage (TES) System

modeling and prediction is made feasible by a relatively simple modification of the proposed modeling and computational approach described here.

First, the basic physics and the associated mathematical model for the unsteady melting problem depicted in Fig. 2.2 is discussed in this section. Next in subsequent sections, a complete computational simulation procedure for solving the unsteady charging problem is presented. The HTF flow is hydro-dynamically steady and its temperature $T_{i n}$ at the inlet of the tube is also steady or constant. The HTF flow initiates an unsteady (time dependent) temperature variations at the wall, within the HTF, and within the PCM. To model the unsteady problem, some suitable initial condition is assumed for $t \leq 0$. As interest is in the solution for large times $t>t^{*}$ (where $t^{*}$ is the time by which a very thin layer of melted PCM encapsulates the tube), any reasonable choice for initial conditions will suffice. Therefore, for $t \leq 0$, the steady HTF flow in the pipe is assumed to be realized under conditions of an encapsulating heat-sink (which has infinite specific heat and thermal conductivity) at uniform temperature $T_{\infty}$. This temperature $T_{\infty}$ is also the far field temperature of the PCM under actual operating conditions. At $t=0$, the heat sink is removed and replaced by the actual PCM (which has a finite specific heat and 


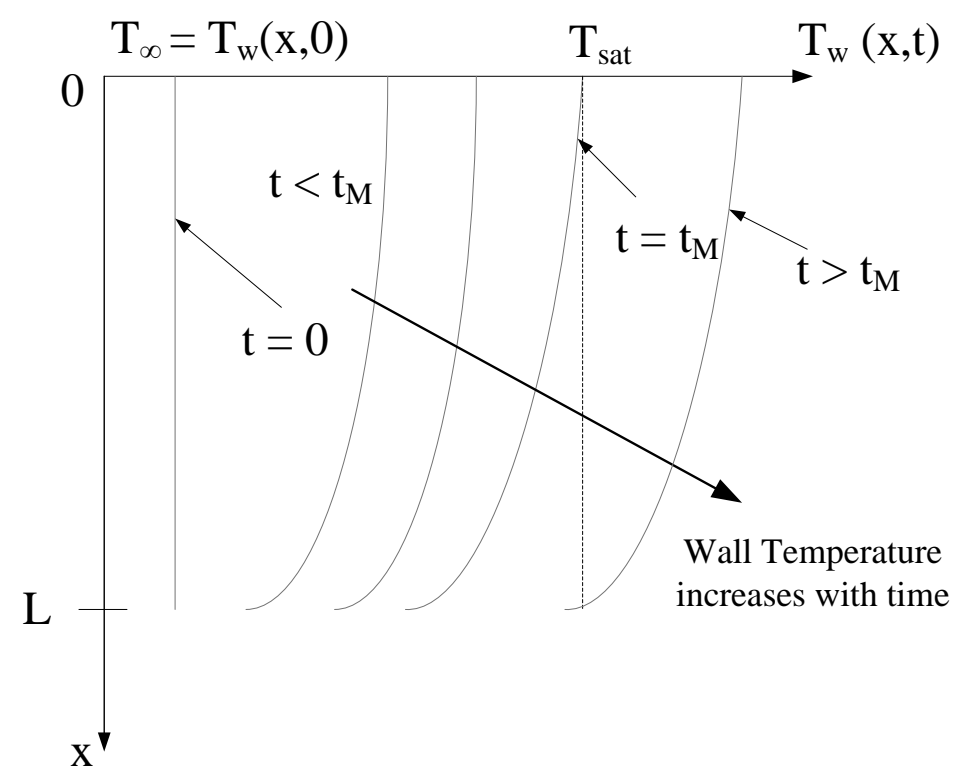

Figure 2.3 : Schematic of Wall Temperature $T_{w}(x, t)$ versus downstream distance $x$

a finite thermal conductivity), whose far field temperature is same as the assumed initial temperature $T_{\infty}$.

The two time durations of interest are: (i) a "before melting" period of $0 \leq t \leq t_{M}$, and (ii) a "melting" period of $\geq t_{M}$. The manner in which the tube's inner (and outer) surface temperature varies with time is of interest. As a simplifying approximation (which can be easily relaxed), the tube thickness is considered negligible. The inner and outer pipe wall temperatures are equal and denoted (see Fig. 2.2) as $T_{w}(x, t)$. The spatial and time variations of wall temperature $T_{w}(x, t)$ and wall heat flux $q^{\prime \prime}{ }_{w}(x, t)$ are important unknown variables which need to be determined. At any time $t$, an expected qualitative variation of wall temperature $T_{w}(x, t)$ with $\mathrm{x}$ is depicted in Fig. 2.3. At $t=$ $t_{M}$, the tube wall temperature $T_{w}(x, t)$ first reaches the saturation temperature $T_{\text {sat }}$ at $x=0$ (see point $M$ in Fig. 2.2), i.e. $T_{w}\left(0, t_{M}\right)=T_{\text {sat }}$ (see Fig. 2.3). Other important objectives of the unsteady simulation for the problem in Fig. 2.2 are to determine wall heat flux $q^{\prime \prime}{ }_{w}(x, t)$ and time varying location of the melt-solid interface (see I ( $\mathrm{t}$ ) in Fig. 2.2). For the axis-symmetric simulation, the melt-solid interface in Fig. 2.4 is defined by $r=\Delta(x, t)$. 


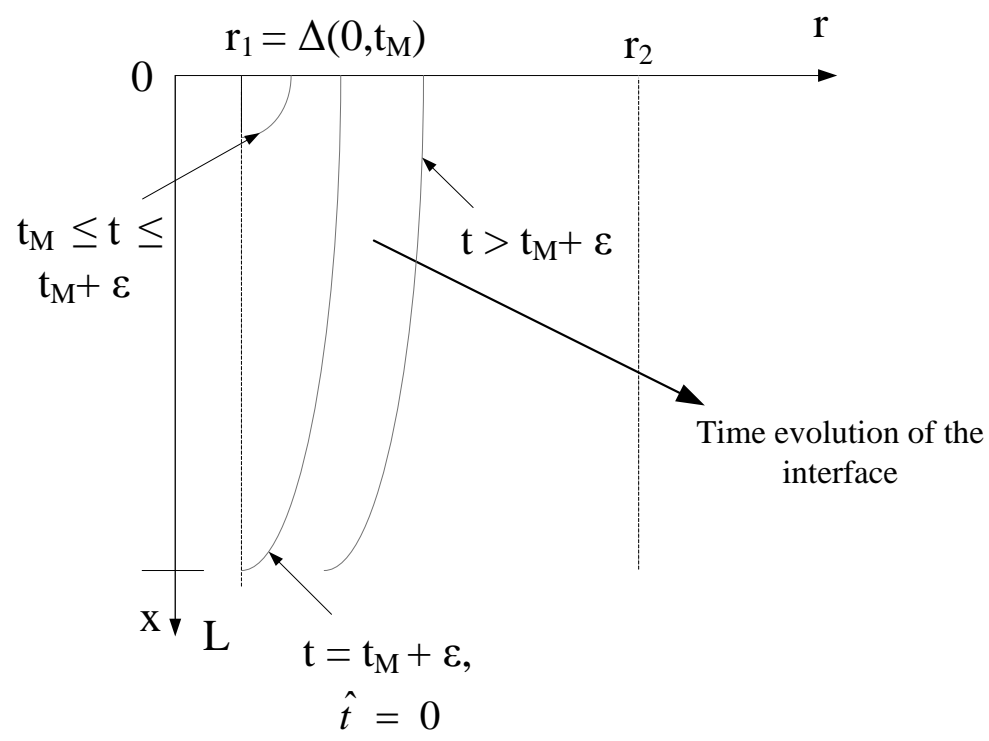

Figure 2.4: Schematic Evolution of Interface $\Delta(x, t)$ versus Downstream Distance $x$

In addition to the desired results depicted in Figs. 2.3 -2.4, other quantities of interest are listed below:

(i) The total supplied heat $Q_{\left[t_{M}, t\right]}$ made available at the pipe wall as a function of $t$, where

$$
Q_{\left[t_{M}, t\right]}=\int_{t_{M}}^{t} \int_{0}^{L} q^{\prime \prime}{ }_{w}(x, \tau) \mathrm{dx} \cdot \mathrm{d} \tau
$$

(ii) The total supplied heat $Q_{p i p e-i n}\left[t_{M}, t\right]$ as a function of $t$ made available at the HTF pipe inlet and defined as:

$$
Q_{\text {pipe-in }\left[t_{M}, t\right]}=\dot{M}_{\text {in }} * C_{P_{F}} * T_{\text {in }} *\left(t-t_{M}\right)
$$

(iii) The total heat stored in the PCM $Q_{[0, T]}^{\text {stored }}$ given as

$$
Q_{\left[t_{M}, t\right]}^{\text {stored }}=M_{\text {melt }}(t) * h_{s f}
$$

where $M_{\text {melt }}(t)$ is the mass of molten liquid at time $\mathrm{t}$ and $h_{s f}$ is a representative value of the latent-heat of fusion 
(iv) The nature of temperature variations $T_{1}(x, r, t)$ and $T_{2}(x, r, t)$ in the molten and solid PCM.

(v) The time-varying nature of the effectiveness $\epsilon_{1}(t)$ and $\epsilon_{2}(t)$ of the thermal storage systems as a function of $t>t_{M}$, length $\mathrm{L}$ and the nature of the thermal boundary condition at $r=r_{2}$ and defined as:

$$
\epsilon_{1}(t) \equiv \frac{Q_{\left[t_{M}, t\right]}^{\text {stored }}}{Q_{\text {pipe-in }\left[t_{M}, t\right]}} \text { and } \epsilon_{2}(t) \equiv \frac{Q_{\left[t_{M}, t\right]}^{\text {stored }}}{Q_{\left[t_{M}, t\right]}}
$$

(vi) The time-varying nature of the efficiency $\eta(t)$ of the thermal energy storage process defined as:

$$
\eta(t) \equiv \frac{Q_{\left[t_{M}, t\right]}^{\text {stored }}}{\left.W_{\text {pump }}\right|_{\left[t_{M}, t\right]}}
$$

Where $\left.W_{\text {pump }}\right|_{\left[t_{M}, t\right]}=\left(\frac{\dot{M}}{\rho_{F}}\right) * \Delta p *\left(t-t_{M}\right)$ is the mechanical work done over the duration of interest. 


\section{Interface Conditions}

\subsection{Physics at the Melt-Solid Interface}

As melting progresses, the interface between the solid and the liquid moves and latent heat is released by the solid. At the interface, under the assumption of equilibrium thermodynamics and nearly constant pressure, the temperatures across the interface are continuous and nearly constant at a value of $T_{\text {sat }}$. The conservation of energy is next described with the help of Fig. 3.1. If $\left.q^{\prime \prime}{ }_{1}\right|^{\text {int }}$ is the amount of heat flux which arrives (from liquid phase) at a point on the interface, part of this heat flux is utilized to melt the solid and the remaining heat flux $\left.q_{2}{ }_{2}\right|^{\text {int }}$ leaves that point on the interface towards the solid PCM.

Therefore, the difference between these two heat fluxes equals the mass rate of melting $(\dot{m})$ times the latent heat of phase change $\left(h_{s f}\right)$. That is:

$$
q_{1}^{\prime \prime i n t}-q_{2}^{\prime \prime i n t}=\dot{m} * h_{s f}
$$

The above key interface condition along with the remaining melt-solid interface conditions are rigorously summarized in Appendix C.

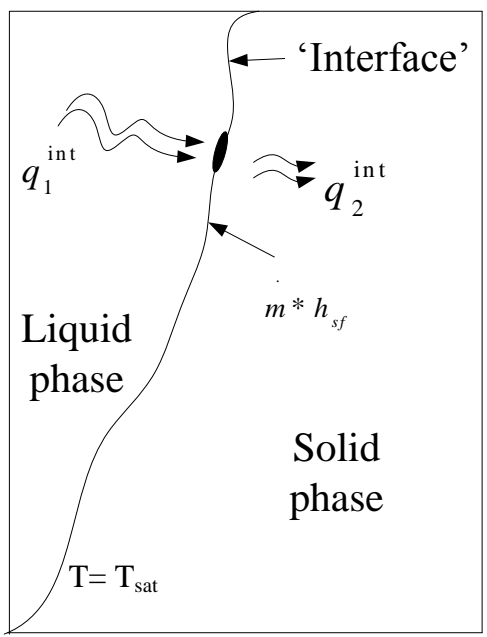

Figure 3.1:Melting of a Material 


\subsection{Physics at the HTF - Pipe - PCM interface}

As pipe wall thickness is neglected, the fluid-pipe wall interface and the pipe wall-PCM interface coalesce to a single interface. At this interface, the continuity of temperature and heat flux must hold. This is expressed as

$$
\begin{aligned}
& \left.T_{\text {pipe }}\right|_{\text {inner }}=\left.T_{\text {pipe }}\right|_{\text {outer }}=T_{w}(x, t) \\
& \text { and }\left.\quad q^{\prime \prime}{ }_{\text {pipe }}\right|_{\text {inner }}=\left.q^{\prime \prime}{ }_{\text {pipe }}\right|_{\text {outer }}=q^{\prime \prime}{ }_{w}(x, t)
\end{aligned}
$$

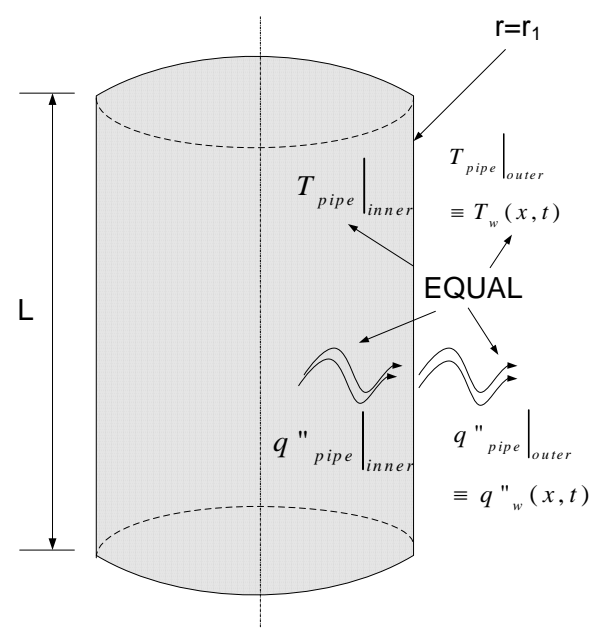

Figure 3.2: Conjugate Heat Transfer Problems for the LHTES System 


\section{Conjugate Heat Transfer Problems for the LHTES System}

In this section, the nature of the conjugate heat transfer problems and their respective spatial domains for the PCM and the HTF flow sub-system (shown in Fig. 2.2) are described. The governing equations, initial conditions and boundary conditions used to solve the PCM and the HTF flow problems over different time durations of interest are described here.

\subsection{Nature of Conjugate Heat Transfer Problems and their respective Domains}

The conjugate heat transfer problems of interest are best described for three separate time durations, namely

(i) "Before melting" duration of $0 \leq t \leq t_{M}$. For this, the problem schematic is shown in Fig. 4.1a.

(ii) Rapid movement of melt-solid interface over a very short interval of time $\left(t_{M} \leq t \leq t_{M}+\varepsilon \equiv t^{*}\right.$, where $\varepsilon$ is very small number).

(iii) "Melting" duration of $t \geq t^{*} \equiv t_{M}+\varepsilon$. For this duration, the problem schematic is shown in Fig. 4.1b.

The rapidly moving nature of the melting over duration $t_{M} \leq t \leq t_{M}+\varepsilon$ is not central to the problem at hand and the reasons for the smallness of its duration $(\varepsilon)$ are discussed in Appendix B.

For $0 \leq t \leq t_{M}$, the conjugate problems in Fig. 4.1a consists of the following domains:

a) The HTF pipe flow problem defined over the domain $0 \leq r \leq r_{1}$ and $0 \leq x \leq$ $L$

b) The unsteady conduction problem for the PCM defined over the domain $0 \leq$ $x \leq L$ and $r_{1} \leq r \leq r_{2}$

For $t \geq t^{*} \equiv t_{M}+\varepsilon$, the conjugate problem in Fig. 4.1b consists of the following domains: 


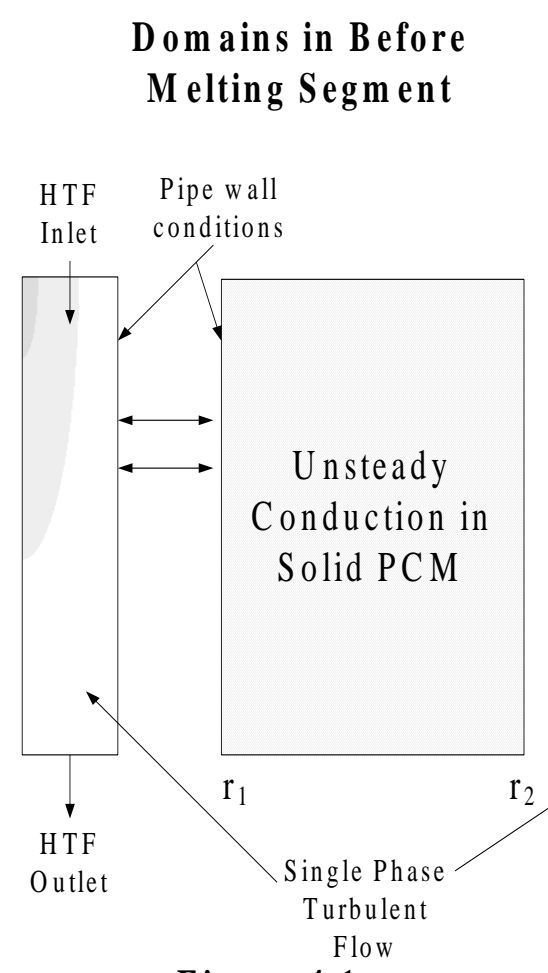

Figure 4.1 a

\section{Dom ains in After \\ Melting Segment}

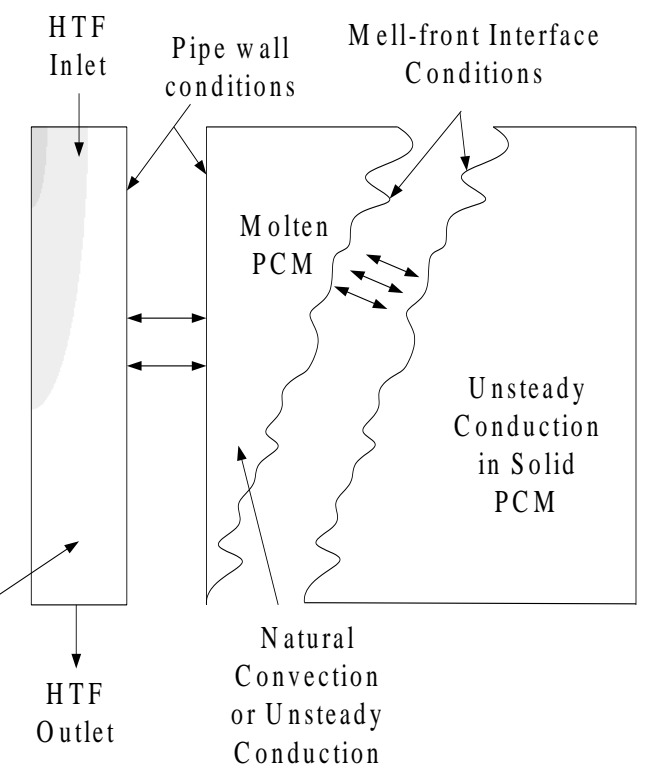

Figure $4.2 \mathrm{~b}$

Figure 4.1: Domains for Conjugate Heat Transfer Problem

a) The HTF pipe flow problem defined over the domain $0 \leq x \leq L$ and $r_{1} \leq r \leq$ $r_{2}$

b) The molten PCM problem modeled either as conduction for stationary melt or a natural convection problem for the melt is defined for the domain $0 \leq x \leq L$ and $r_{1} \leq r \leq \Delta(x, t)$

c) The unsteady conduction problem for the solid PCM is defined for the domain $0 \leq x \leq L$ and $\Delta(x, t) \leq r \leq r_{2}$.

Since the HTF problem remains the same for both "before melting" and "melting" durations, it is described first. 


\subsection{HTF flow in a pipe}

The turbulent HTF flow in pipe for all times can be formulated as a 'forced' convection heat transfer problem.

As the flow is hydro-dynamically steady, the unsteady mean temperature variations for $t>0$, can be shown to arise from the unsteady pipe wall temperature $T_{w}(x, t)$ and all unsteady terms in the governing energy equation for the HTF flow can be ignored (see Appendix A). The unsteadiness in the pipe wall temperature conditions can be easily accommodated by the qua-steady flow and explained next. This simplification is possible because the characteristic time $\Delta t_{c-H T F}$ associated with the flow is much smaller than the characteristic time $\left(\Delta t_{c_{1}}=\Delta \tau * \frac{l_{c}^{2}}{\alpha}\right)$ associated with the changes in wall temperature $T_{w}(x, t)$.

The characteristic time (see Appendix A for more details) for the wall temperature $T_{w}(x, t)$ is same as the characteristic time for the unsteady temperature variations within the PCM (for $0 \leq t \leq t_{M}$ as well as for $t \geq t_{M}$ ). As a result, the quasi-steady forced

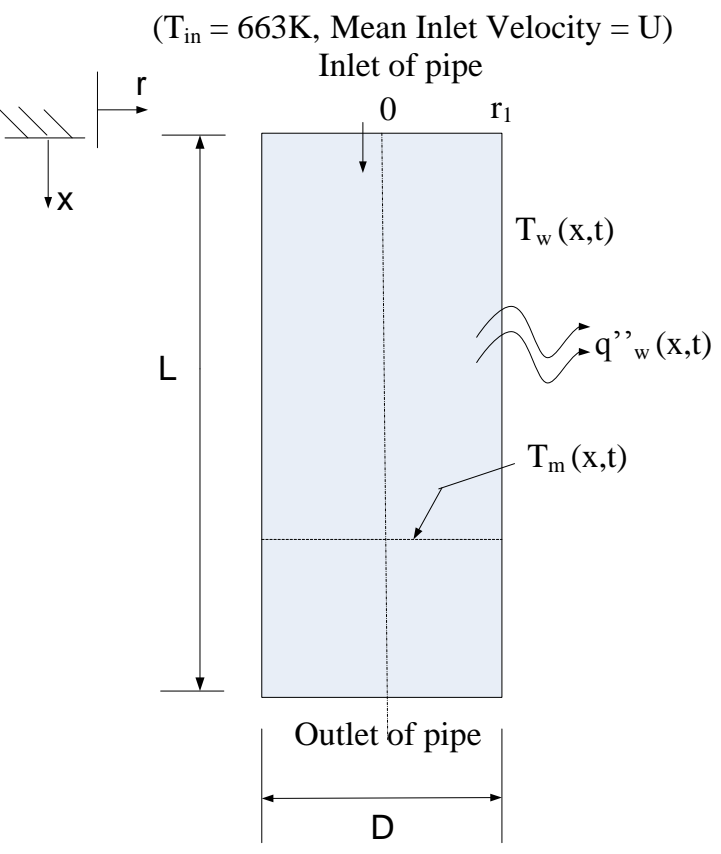

Figure 4.2: HTF Flow in Pipe 
convection turbulent flow problem for the HTF flow reduces to the well known (Incropera, 2006) one-dimensional results given below.

Using well known terminology (see (Incropera 2006)) for forced convection in a pipe, we denote the bulk mean HTF temperature by $T_{m}(x, t)$, the local heat transfer coefficient by $h_{x}$ and the local wall heat flux by $q^{\prime \prime}{ }_{w}(x, t)$. Then well known results and definitions (Incropera 2006) are:

i) The wall heat flux $q^{\prime \prime}{ }_{w}(x, t)$ in Fig. 4.2 is given by

$$
q^{\prime \prime}(x, t)=h_{x}\left[T_{m}(x, t)-T_{w}(x, t)\right]
$$

ii) Utilizing the above definition of wall heat flux and incorporating it in the energy balance of the HTF flow for a differential length $\Delta x$ of the pipe (see Fig. 4.2) yields (Incropera 2006)

$$
\frac{d T_{m}}{d x}=\frac{\pi D h_{x}}{\dot{m} * C_{P}}\left[T_{m}(x, t)-T_{w}(x, t)\right]
$$

In the above and subsequent analysis of the HTF flows, the HTF fluid properties of density, specific heat, viscosity and the thermal conductivity are modeled by their representative constant values and denoted as $\rho_{F}, C_{P_{F}}, \mu_{F}$ and $k_{F}$ respectively.

iii) For most of the pipe, except for a short distance from the inlet (Incropera 2006) fully developed turbulent flow conditions can be assumed. Therefore, the local heat transfer coefficient $h_{x}$ is given by

$$
N u_{D} \equiv \frac{h_{x} D}{k}=0.023 * R e_{D}^{4 / 5} * \operatorname{Pr}^{0.3}
$$

where $N u_{D}=\rho_{F *} U * \frac{D}{\mu_{F}}, \operatorname{Pr}=\mu_{F} * C_{p_{F}} / k_{F}$

iv) For developing turbulent flow conditions near $\mathrm{x}=0, h_{x}$ is given by 


$$
\begin{aligned}
N u_{D} \equiv \frac{h_{x} D}{k} & \\
& =0.012 *\left(\operatorname{Re}_{D}{ }^{0.87}-280\right) * \operatorname{Pr}^{0.3} \\
& *\left[1+\left(\frac{D}{x}\right)^{2 / 3}\right]
\end{aligned}
$$

\subsection{Other Conjugate Heat Transfer Problems for "Before Melting" Duration}

For $0 \leq t \leq t_{M}$, ("before melting") time duration, two spatial domains to be considered are: (i) for HTF flow in the pipe, and (ii) The solid PCM. The HTF flow problem is same as described in section 4.2 .

\subsubsection{Unsteady Conduction for the Solid PCM}

For $0 \leq t \leq t_{M}$, as shown in Fig. 4.1a, there is no molten PCM. Unsteady conduction problem for the solid PCM with initial and boundary conditions are schematically shown in Fig. 4.3. The density, specific heat and thermal conductivity of the solid PCM are represented by their representative constant of $\rho_{2}, C_{P_{2}}$ and $k_{2}$ respectively.

The differential form of the energy equation (Incropera 2006) for the interior of the solid $\mathrm{PCM}$ is the governing equation. This is given as (for cylindrical coordinates)

$$
\frac{1}{r} \frac{\partial}{\partial r}\left(r \frac{\partial T_{2}}{\partial r}\right)+\frac{\partial^{2} T_{2}}{\partial z^{2}}=\frac{\rho_{2} C_{p_{2}}}{k_{2}} \frac{\partial T_{2}}{\partial t}
$$

The resulting problem is parabolic in nature. Therefore, the initial conditions (at $t=0$ ) together with the boundary conditions at $r=r_{1}, r=r_{2}, x=0$, and $x=L$ are sufficient for a computational solution of the problem.

The boundary conditions are:
i) $T\left(x, r_{2}, t\right)=T_{\infty}$ for $0 \leq x \leq L$
ii) $q^{\prime \prime}(0, r, t)=0$ for $r_{1} \leq r \leq r_{2}$ 


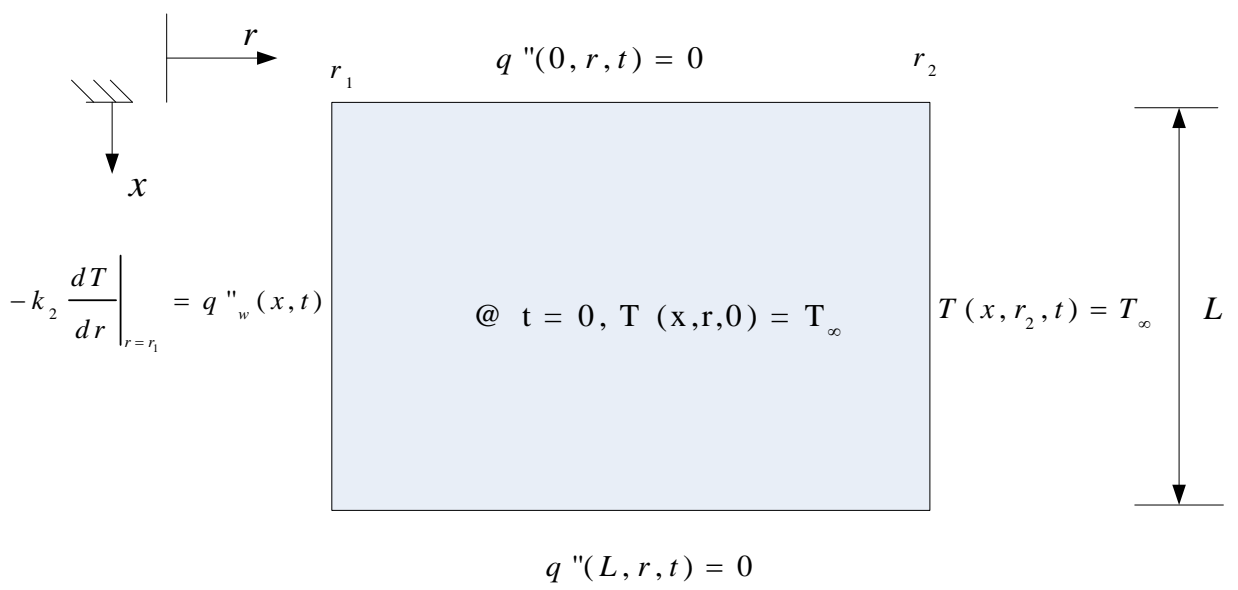

Figure 4.3: Initial and Boundary Conditions for Solid PCM domain

iii) $-\left.k_{2} \frac{\partial T_{2}}{\partial r}\right|_{r=r_{1}}=q^{\prime \prime}{ }_{w}(x, t)$ for $0 \leq x \leq L$, where the wall heat flux $q^{\prime \prime}{ }_{w}(x, t) \equiv$ $q^{\prime \prime}\left(x, r_{1}, t\right)$ is obtained from the conjugate analysis procedure described later in section 5.1.

iv) $q^{\prime \prime}(L, r, t)=0$ for $r_{1} \leq r \leq r_{2}$

The initial condition is

$$
\mathrm{T}(r, x, 0)=T_{\infty} \text { for all } x \text { and } r \text { within the PCM. }
$$

After suitable discretization of the time and spatial domains, the above problem is computationally solved on COMSOL. The solution is marched forward in uniform time steps $\Delta t$. Thus solutions are available at discrete times $t=n * \Delta t$, where $n=$ $1,2,3, \ldots, \Delta t \equiv 0.025 * \Delta t_{c 1}$, and $\Delta t_{c 1} \equiv \frac{\left(r_{2}-r_{1}\right)^{2}}{\alpha_{2}}$ is the characteristic time for unsteady conduction in the solid PCM.

\subsection{Other Conjugate Heat Transfer Problems for the "Melting" Duration}

For "melting" time duration $\left(t \geq t_{M}+\varepsilon\right)$, three spatial domains namely HTF flow domain in the pipe, the molten PCM domain, and the solid PCM domain are present. Since the HTF flow problem has already been described in section 4.2, the governing equations and the boundary conditions for the molten PCM and the solid PCM are described in this section. 


\subsubsection{Unsteady Conduction Problem for the Molten PCM}

Though a better formulation for the nearly stagnant molten PCM problem is one in which natural convection is taken into account, natural convection is ignored in the present study and the problem is modeled as if unsteady heat conduction is the dominant heat transfer mechanism. Under this approximation, the problem is governed by the following equation:

GE:

$$
\frac{1}{r} \frac{\partial}{\partial r}\left(r \frac{\partial T_{1}}{\partial r}\right)+\frac{\partial^{2} T_{1}}{\partial x^{2}}=\frac{\rho_{1} C_{P_{1}}}{k_{1}} \frac{\partial T_{1}}{\partial t}
$$

(Heat Equation in a cylindrical coordinates)

Boundary Conditions shown in Fig. 4.4 are as follows:

i) $\quad T\left(x, r_{1}, t\right) \equiv T_{w}(x, t)$ for $0 \leq x \leq L$, where the wall temperature $T_{w}(x, t)$ is obtained from the conjugate analysis procedure described later in section 5.2.

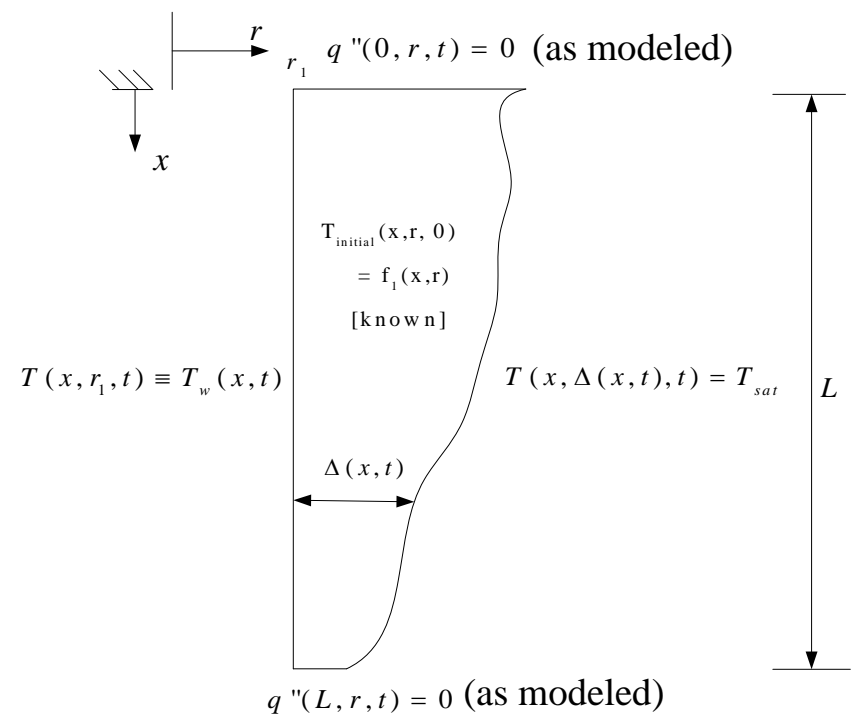

Figure 4.4: Initial and Boundary Conditions for Molten PCM domain

ii) $\quad T(\Delta, x, t)=T_{\text {sat }}$ for $0 \leq x \leq L$

iii) $\quad q^{\prime \prime}(0, r, t)=0$ for $r_{1} \leq r \leq \Delta(x, t)$

iv) $\quad q^{\prime \prime}(L, r, t)=0$ for $r_{1} \leq r \leq \Delta(x, t)$ 
Initial Condition:

$$
T_{\text {initial }}=T(x, r, t=0)=f_{1}(r, x)(\text { known })
$$

After suitable definition and updating procedure for the unknown interface location $\Delta(x, t)$ as described later on, the time and spatial domains are suitable discretized for a computational solution on COMSOL. The solution is marched forward in uniform time steps $\Delta t$. Thus solutions are available at discrete times $t=n * \Delta t$, where $n=$

$1,2,3, \ldots, \Delta t \equiv 0.0125 * \Delta t_{c 1}$, and $\Delta t_{c 1} \equiv \frac{\left(r_{2}-r_{1}\right)^{2}}{\alpha_{2}}$ is the characteristic time for unsteady conduction in the solid PCM.

\subsubsection{Unsteady Conduction Problem for the Solid PCM on the Other Side of the Melt-front}

For $t \geq t_{M}$, the problem formulation for the PCM on the other side of the melt-front is similar to the one described for the "before melting" duration. The key difference is that the left boundary @ $r=r_{1}$, is replaced by the unknown interface location $r=\Delta(x, t)$ shown in Fig. 4.5. A suitable definition and updating procedure for the unknown interface location $\Delta(x, t)$ is described later on.

Again, transient conduction within the solid PCM for "melting" duration is governed by the same equation as for transient conduction within solid PCM for "before melting" duration. It is expressed as:

$$
\frac{1}{r} \frac{\partial}{\partial r}\left(r \frac{\partial T_{2}}{\partial r}\right)+\frac{\partial^{2} T_{2}}{\partial z^{2}}=\frac{\rho_{2} C_{p_{2}}}{k_{2}} \frac{\partial T_{2}}{\partial t}
$$

(Heat Equation in a cylindrical coordinates) 


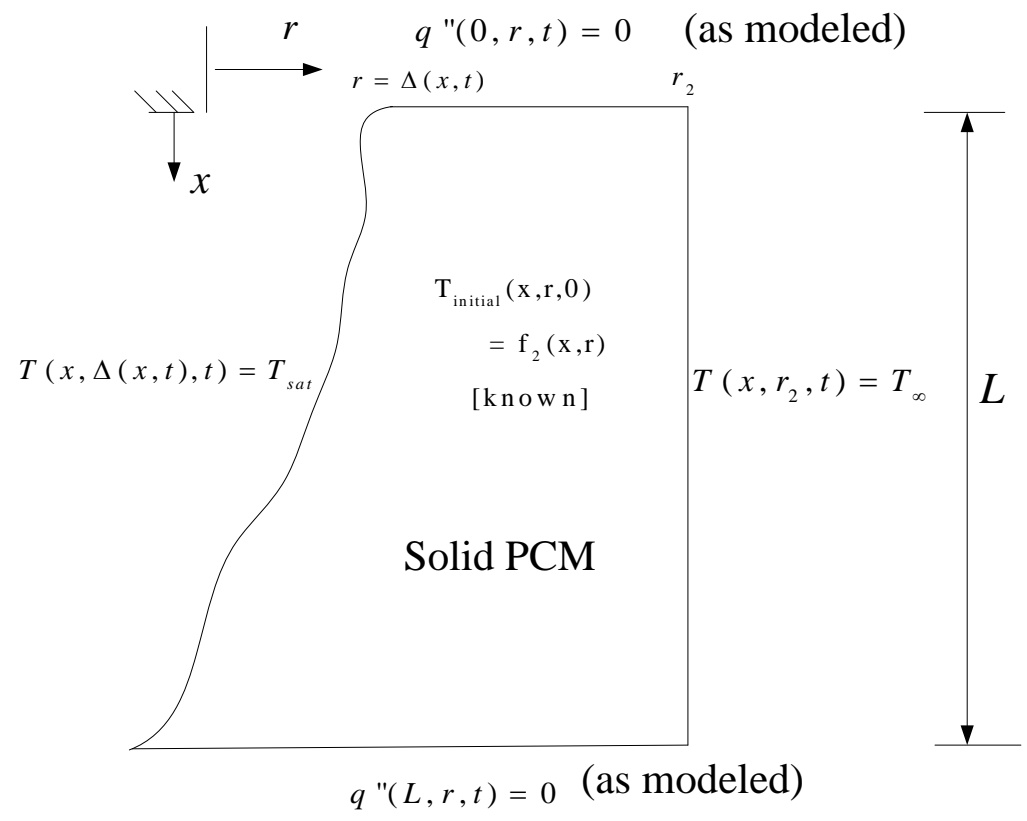

Figure 4.5: Initial and boundary conditions for solid PCM domain

Boundary Conditions:

i) $\quad T(x, \Delta, t)=T_{\text {sat }}$ for $0 \leq x \leq L$

ii) $\quad T\left(x, r_{2}, t\right)=T_{\infty}$ for $0 \leq x \leq L$

iii) $\quad q^{\prime \prime}(0, r, t)=0$ for $\Delta(x, t) \leq r \leq r_{2}$

iv) $\quad q^{\prime \prime}(L, r, t)=0$ for $\Delta(x, t) \leq r \leq r_{2}$

Initial Condition:

$T_{\text {initial }}=T(x, r, t=0)=f_{2}(r, x)($ known $)$

After suitable discretization of the time and spatial domains, the above problem is computationally solved on COMSOL. The solution is marched forward in uniform time steps $\Delta t$. Thus solutions are available at discrete times $t=n * \Delta t$, where $n=$ $1,2,3, \ldots, \Delta t \equiv 0.0125 * \Delta t_{c 1}$, and $\Delta t_{c 1} \equiv \frac{\left(r_{2}-r_{1}\right)^{2}}{\alpha_{2}}$ is the characteristic time for unsteady conduction in the solid PCM. 


\section{The Solution Approach for the Conjugate Problems}

It is assumed that, initially $(t \leq 0)$, the HTF pipe is in contact with perfect heat sink at temperature $T_{\infty}$. This temperature $T_{\infty}$ is the same as far-field temperature $r=r_{2}$. As the HTF steadily flows through the inlet of the pipe at temperature $T_{i n}$, its mean temperature drops with downstream distance. The assumed presence of the heat sink allows wall temperature to be $T_{w}(x, t)=T_{\infty}$ for all $t \leq 0$. This allows the values of wall heat flux $q^{\prime \prime}{ }_{w}(x, 0)$ and mean HTF temperature variation $T_{m}(x, 0)$ to be calculated with the help of equations described in section 4.2.

\subsection{Numerical Solution Approach for the "Before Melting" Duration Conjugate Problems}

As explained earlier, for $0 \leq t \leq t_{M}$ " - the before melting" duration, there are only two spatial domains. These are the HTF flow domain in the pipe and the solid PCM domain outside the pipe. Numerical solution approach for the "before melting" duration is as follows:

a) For $t \leq 0$, pipe is in contact with perfect heat sink at temperature $T_{\infty}$. Thus wall heat flux $q^{\prime \prime}{ }_{w}(x, t=0)$, wall temperature $T_{\infty}$, and the mean fluid temperature $T_{m}(x, t=0)$ are known from the steady HTF problem solution discussed in section 4.2 .

b) For $t=\Delta t$, solve the unsteady conduction problem for the solid PCM (as in section 4.3.1) with $T_{\text {initial }}=T(x, r, t=0)=T_{\infty}$ and obtain the wall temperature $T_{w}(x, t+\Delta t)$ as part of the solution.

c) Next for $t=\Delta t$, the quasi-steady response of the HTF flow is assessed for the new wall temperature $T_{w}(x, t+\Delta t)$ obtained in the previous step. For assessment, solve the turbulent HTF flow problem (as in section 4.2) to obtain the new mean fluid temperature $T_{m}(x, t+\Delta t)$ and the new wall heat flux $q^{\prime \prime}{ }_{w}(x, t+\Delta t)$.

d) Repeat steps $\mathrm{b}$ and $\mathrm{c}$ till converged values of $T_{w}(x, t+\Delta t)$ and $q^{\prime \prime}{ }_{w}(x, t+\Delta t)$ are obtained.

e) With converged values of $T_{w}(x, t+\Delta t), q^{\prime \prime}{ }_{w}(x, t+\Delta t)$ and updated value of the initial temperature for the interior of the solid PCM (i.e. the converged values of 
$T(x, r, t+\Delta t)$ replacing $T(x, r, 0)$ in Fig. 4.3) repeat steps $\mathrm{b}, \mathrm{c}$ and $\mathrm{d}$ to obtain the solution for the next time-stept $=2 * \Delta t$.

f) Repeat the above steps (b) - (e) until $t=t_{M}=N * \Delta t$ is reached. Recall $t_{M}$ is the time where the wall temperature at $x=0$ reaches the melting point temperature value of $T_{\text {sat }}$.

At this time $t=t_{M}$, the interface is merely a point M in Fig. 2.2. Subsequently, after a very small time duration $\varepsilon$, the interface unfolds rapidly (see Appendix B) to cover the length of the pipe as a thin encapsulating interface (see interface location in Fig. 2.4 at $t=t_{M}+\varepsilon$ ). The underlying reason for this phenomenon (modeled in Appendix B) is that both the inlet and outlet temperatures at time $t=t_{M}$ (namely $T_{\text {in }}$ at the inlet and $T_{m}\left(L, t_{M}\right)$ at the exit) are significantly larger than the melting temperature $T_{\text {sat }}$. This, together with the fact that thermal inertial of thin melt zone is negligible, makes the encapsulation duration $\varepsilon$ very small. Complex simulations are required to capture this movement of the interface with the help of very small timesteps. Since this movement of the interface is not important for the problem under consideration, following an analytical order of magnitude estimate of $\varepsilon$ in Appendix $\mathrm{B}$, this time duration is neglected here. As a result, an arbitrary profile (which encapsulates the pipe and is very thin) for the interface location is assumed for $t=$ $t_{M}+\varepsilon$. This arbitrary profile of interface is then corrected by the numerical solution approach for the "melting" duration described in the next section. It is important to recognize that the assumed/corrected interface location for $t=t_{M}+\varepsilon$ does not affect the predictions for the times $t\left(\gg t_{M}+\varepsilon\right)$ of interest here.

\subsection{Numerical Solution Approach for the "Melting" Duration Conjugate Problems}

As explained earlier, for $t \geq t_{M}+\varepsilon$, the "melting" duration has three spatial domains. These are: (i) the HTF flow domain in the pipe, (ii) the molten PCM domain, and (iii) the solid PCM on the other side of the melt-front. For this algorithm, a shifted time variable namely $\hat{t} \equiv t-\left(t_{M}+\varepsilon\right)$, is used to keep track of the subsequent times $\hat{t}$ of interest. Note 
that $\hat{t}=0$, is same as $t=\left(t_{M}+\varepsilon\right)$. Numerical solution approach for the "melting" duration is as follows:

a) Start with $T_{w}(x, \hat{t}=0)$ and the assumed form of $\Delta(x, \hat{t}=0)=\Delta(x, t=$ $\left.\left(t_{M}+\varepsilon\right)\right)$. Furthermore, for each $x$, assume a linear temperature variation (with $r$ ) for the melt temperature $T_{1}(x, r, \hat{t}=0)$. The values at $r=r_{1}$, being $T_{w}(x, \hat{t}=0)$ and at $r=r_{2}$ being $T_{\text {sat }}$. The solid PCM temperature $T_{2}(x, r, \hat{t}=0)$ remains the same as the temperature $T_{2}\left(x, r, t=t_{M}\right)$ obtained for the "before melting" problem at $t=t_{M}$.

b) Starting from $\hat{t}=0$, computationally solve the unsteady conduction problem for the melt to obtain preliminary results for $\hat{t}=\Delta t$. For this, the $\hat{t}=0$ and $\hat{t}=\Delta t$ values for the wall temperature $T_{w}(x, \hat{t})$ and interface location $\Delta(x, \hat{t})$ are assumed to be the same as the ones for $\hat{t}=0$. Furthermore, for advancing the time to $\hat{t}=\Delta t$ the current value of the melt temperature $T_{1}(x, r, \hat{t}=0)$ is also used. From this solution, the $\hat{t}=\Delta t$ values of $\left.k_{1} \frac{\partial T_{1}}{\partial \mathrm{r}}\right|^{\mathrm{i}},\left.k_{1} \frac{\partial T_{1}}{\partial \mathrm{x}}\right|^{\mathrm{i}}$ and $q^{\prime \prime}{ }_{w}(x, \hat{t})$ are obtained.

c) For this location of $\Delta(x, \hat{t}=\Delta t)$, solve the unsteady conduction problem for the solid PCM with the initial solid PCM temperature being the current value of $T_{2}(x, r, \hat{t}=0)$. Once the solution for $\hat{t}=\Delta t$ is available, the computed values of $\left.k_{2} \frac{\partial T_{2}}{\partial \mathrm{r}}\right|^{\mathrm{i}},\left.k_{2} \frac{\partial T_{2}}{\partial \mathrm{x}}\right|^{\mathrm{i}}$ are also obtained.

d) Employing the wall heat flux values of $q^{\prime \prime}{ }_{w}(x, \hat{t})$ as obtained in step (b) for $\hat{t}=$ $\Delta t$, the HTF flow problem solution procedure (described in section 4.2) is used to obtain the mean temperature $T_{m}(x, \hat{t})$ and the wall temperature $T_{w}(x, \hat{t})$ values for $\hat{t}=\Delta t$.

e) Update the value of $\Delta(x, \hat{t}=\Delta t)$ by suitably discretizing and solving the following partial differential equation (see Appendices E - F)

$$
\frac{\partial \Delta}{\partial t}+\frac{\partial \Delta}{\partial x} * \bar{U}=\bar{V}
$$

where $\bar{U}=\frac{1}{\rho_{2} \cdot h s f}\left[-\left.k_{1} \frac{\partial T_{1}}{\partial \mathrm{x}}\right|^{\mathrm{i}}+\left.k_{2} \frac{\partial T_{2}}{\partial \mathrm{x}}\right|^{\mathrm{i}}\right]$ and $\bar{V}=\frac{1}{\rho_{2} \cdot h s f}\left[-\left.k_{1} \frac{\partial T_{1}}{\partial \mathrm{r}}\right|^{\mathrm{i}}+\left.k_{2} \frac{\partial T_{2}}{\partial \mathrm{r}}\right|^{\mathrm{i}}\right]$. 
This partial differential equation (see Appendix D for details on how to solve this equation) arises from the physical requirement that the inter-facial mass flux $\dot{m}$ as obtained from two separate requirements must be the same. These two requirements are: (i) the heat transfer energy balance requirement at the interface, and (ii) the kinematic requirement resulting from the known speed with which the interface moves through the stationary solid PCM.

f) Repeat steps (b) - (e), till converged values of $\Delta(x, \hat{t})$ and $T_{w}(x, \hat{t})$ are obtained for $\hat{t}=\Delta t$.

g) Replacing the $\hat{t}=0$ values in steps (b) - (e) by the $\hat{t}=\Delta t$ values obtained in step (f), steps (b) - (f) are repeated to obtain the values for $\hat{t}=2 * \Delta t$.

h) Repeat above steps (b) - (g) till some suitable $\hat{t}=M * \Delta t$ is reached for which the interface has significantly moved into the solid PCM. 


\section{Convergence and Reliability of the Computed Solution}

\subsection{Grid Independence of the Unsteady Solution}

The above described computational approaches were implemented on COMSOL for two different spatial grid refinements associated with "before melting" and "melting" durations. The two spatial refinements, are schematically depicted at a representative point $\left(x=\frac{L}{2}, r=0.2\right)$ and a representative time in Fig. 6.1 above.

Also, the solution for grid-I above was marched forward in a time with time-step of $\Delta t=\Delta t_{1}$ and for grid-II with time-step of $\Delta t=\Delta t_{2}=\frac{1}{2} \Delta t_{1}$. The converged solutions for a given time " $t=t^{* "}$ were found to be approximately the same and hence the unsteady solutions are considered grid independent. The solutions were tested for girdindependence at various points in the interior of the molten PCM and solid PCM.

For brevity, we only show the results in Fig. 6.2 for the computed variable $\Delta(x, \hat{t})$ as a function of $x$ - once for spatial grid-I and $\Delta t=\Delta t_{1}$ and once for spatial grid-II and $\Delta t=$ $\Delta t_{2}$. In Fig. 6.2, it is seen that for $t=350 \mathrm{~s}$ and $t=700 \mathrm{~s}$ the two solutions are approximately grid independent.

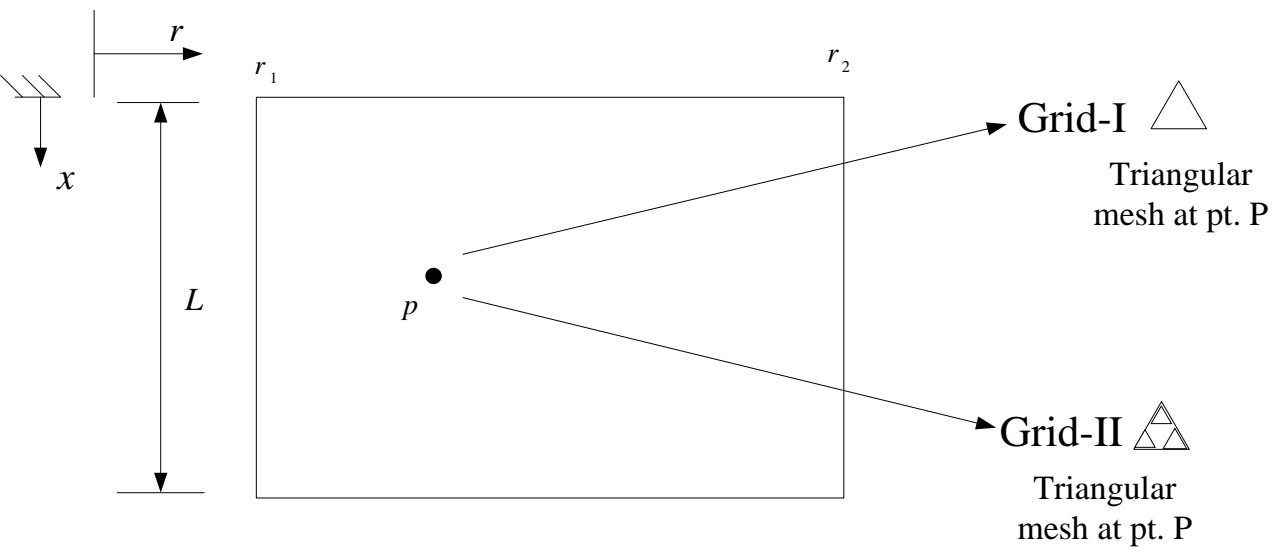

Figure 6.1: Schematic Representation of Spatial Grids Used 


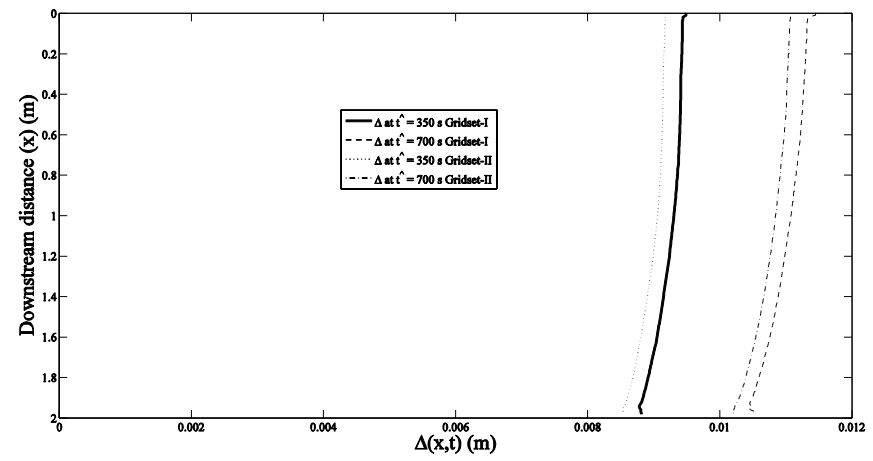

Figure 6.2: Grid Independence for Predicted Location of Interface $\Delta(x, t)$

\subsection{Independence with respect to the Computational Method used for solving the Interface Evolution Equation}

Besides the spatial-temporal grid independence of the unsteady solution obtained for the interior of the molten PCM and the solid PCM, converged the time-evolution of the meltsolid interface may also depend on the computational algorithm used for the numerical solutions of the interface evolution governing equation (one of the interface condition, see equation (C-14) in Appendix C). Two numerical solution approaches (described as method I and II in Appendix D) were used for the interface evolution governing equations towards testing the robustness of the predicted locations of the melt-solid interface. The location of the interface for $t=10000 \mathrm{~s}$ is approximately the same when the above two different numerical solution approaches were used.

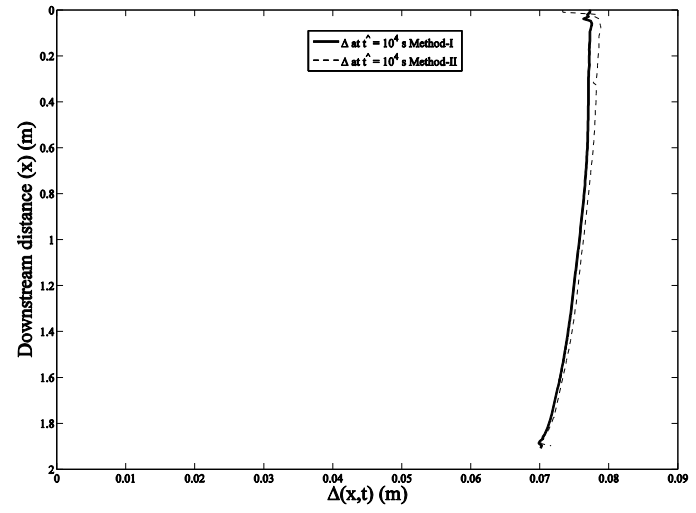

Figure 6.3: Comparison for Location of Interface $\Delta(x, t)$ at $\hat{t}=1 e 4$ 


\subsection{Results for the "Before Melting" Duration}

As described earlier, it is assumed that the pipe is in contact with a perfect heat sink (at uniform temperature $T_{\infty}$ ) for $t \leq 0$. Figure 6.4 shows the plots for the pipe wall temperature $T_{w}(x, 0)$ and the mean HTF temperature $T_{m}(x, 0)$ obtained from the equations described in section 4.2. These conditions of pipe wall temperature $T_{w}(x, 0)$ and the mean HTF temperature $T_{m}(x, 0)$ along with the temperature within the solid PCM $T_{2}(x, r, 0)=T_{\infty}$ are used as initial conditions at $t=0$ for the "before melting" duration.

Using initial conditions shown in Fig. 6.4, the numerical approach for the "before melting" duration (section 5.1) is used to march the solution forward in time. Figure 16 shows converged values of pipe wall temperature $T_{w}\left(x, t=n * \Delta t_{c}\right)$ and the mean HTF temperature $T_{m}\left(x, t=n * \Delta t_{c}\right)$ for $n=0$ and $n=1$. The choice of the time-step $\Delta t_{c}$ involves consideration a several characteristic times present for this LHTES problem (see Appendix A). At $t=0 \mathrm{~s}$, a sudden change is present in the values of the pipe wall temperature $T_{w}(x, t)$ and the mean HTF temperature $T_{m}(x, t)$ is present in Fig. 6.5. This is because, at $t=0 \mathrm{~s}$, the perfect heat sink was removed and replaced by the actual (solid) PCM at uniform temperature of $T_{\infty}$.

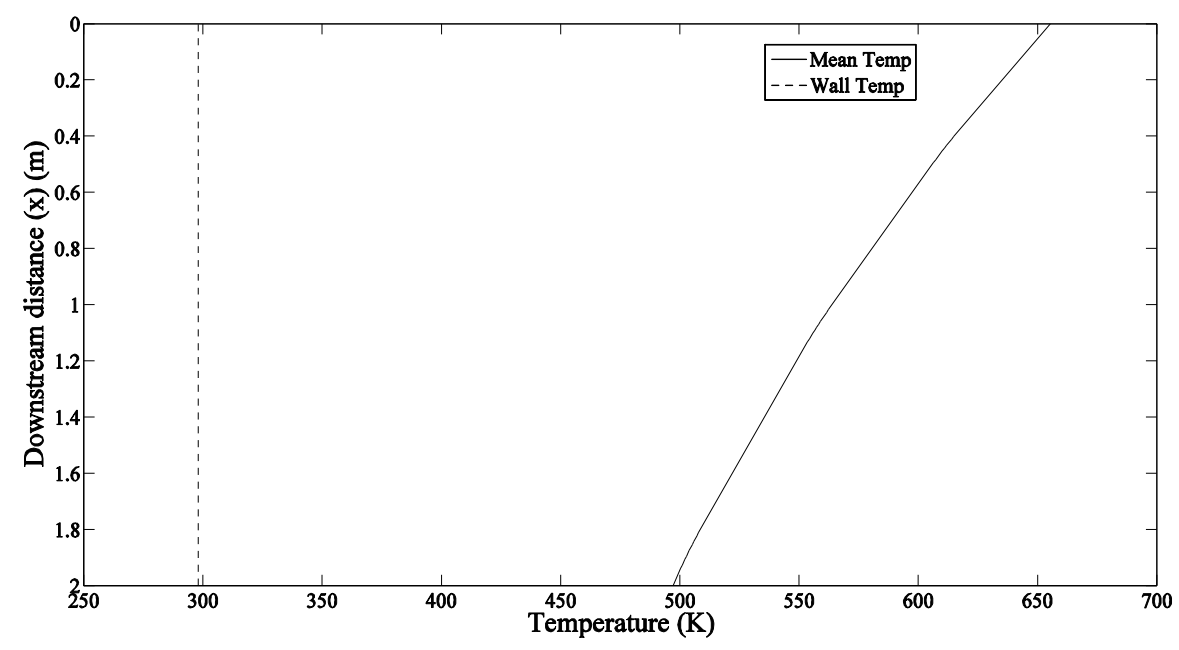

Figure 6.4: Initial Conditions (wall temperature and mean HTF temperature $t=0 \mathrm{~s}$ ) 
Next, simulations for the "before melting" duration are continued until (say $t=t_{M}$ ) wall temperature $T_{w}(x, t)$ reaches the melting temperature $T_{\text {sat }}$ (it will necessary reach this value first at $x=0$ ). Figures 6.6-6.9 show converged values of the wall temperature $T_{w}(x, t)$, the mean HTF temperature $T_{m}(x, t)$, and the pipe wall flux $q^{\prime \prime}{ }_{w}(x, t)$ versus downstream distance $x$ with time (at 50 time-step intervals) as a parameter.

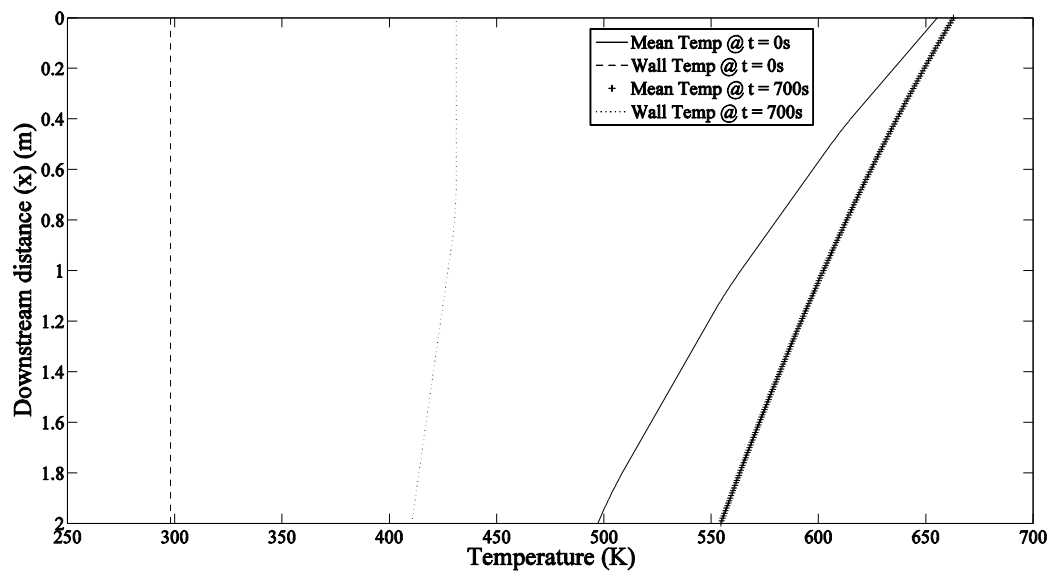

Figure 6.5: Plot Temperature and Mean Temperature (at $t=0 \mathrm{~s}$ and after first time-step)

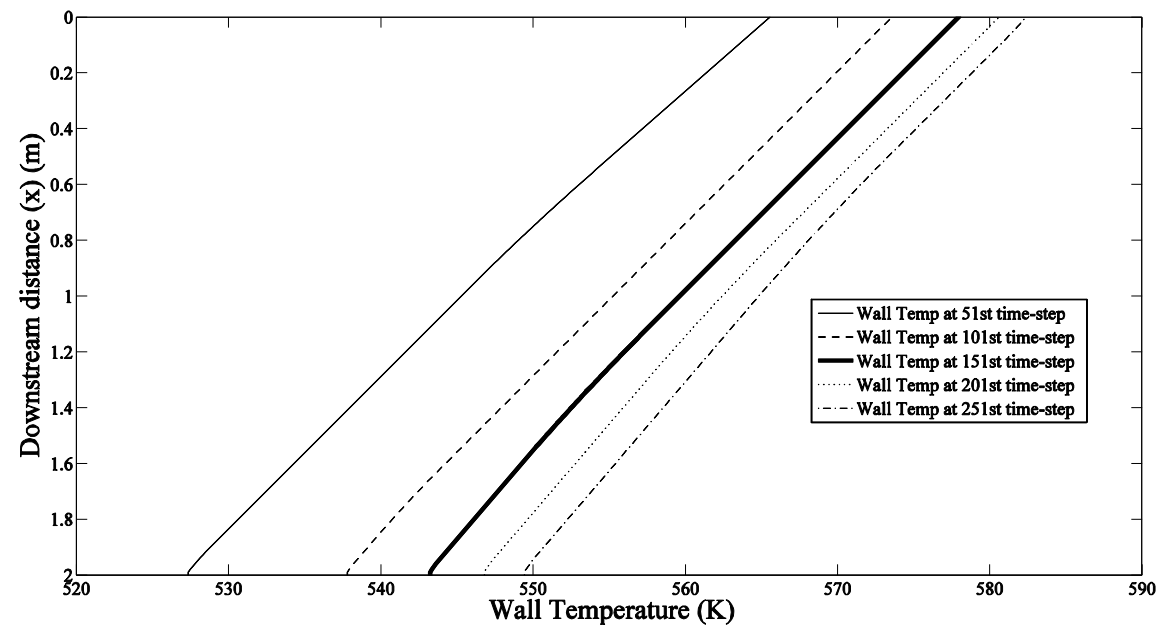

Figure 6.6: Plot of Wall Temperature $T_{w}(x, t)$ after every 50 time-steps 
As wall temperature $T_{w}(x, t)$ increases with time, the driving temperature-difference $\left(\Delta T=T_{\text {in }}-T_{w}(x, t)\right)$, decreases and hence, the heat transferred to the solid PCM as well as the wall flux $q^{\prime \prime}(x, t)$ decreases. This decrease in the wall heat-flux is evident in the plots of Fig. 19. At the end of the $251^{\text {st }}$ time-step, the wall temperature $T_{w}(x, t)$ reaches to the melting temperature $T_{\text {sat }}$ at $x=0$. This time, termed $t=t_{M}=\left(250 * \Delta t_{c}\right)$ indicates an end of the "before melting" duration and appearance of the melt-front as a singular point (see point M in Fig. 2.2).

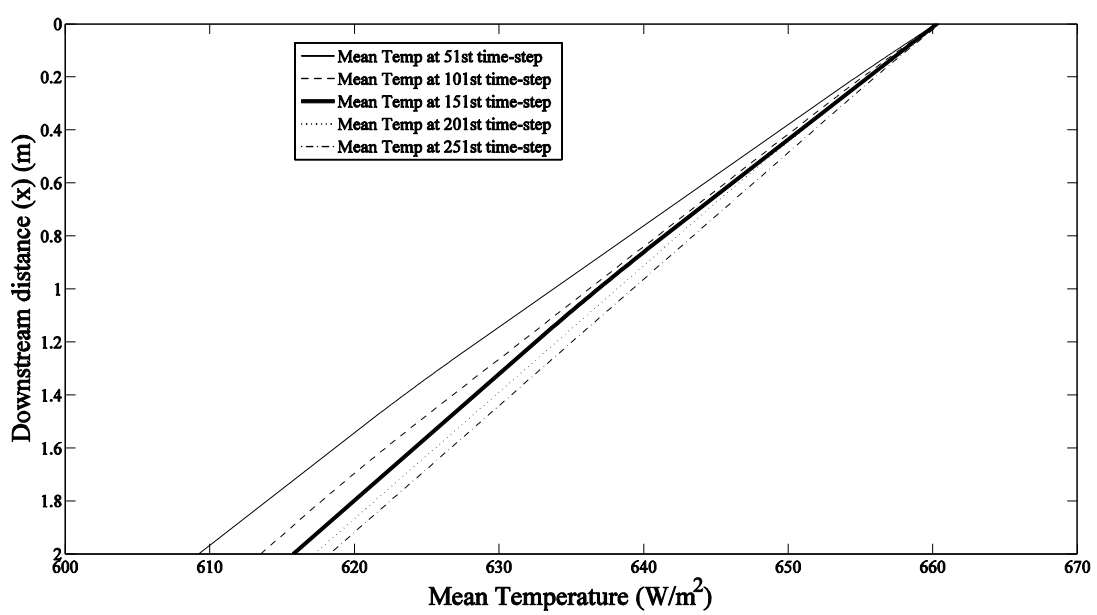

Figure 6.7: Plot of Mean Temperature $T_{m}(x, t)$ after every 50 time-steps

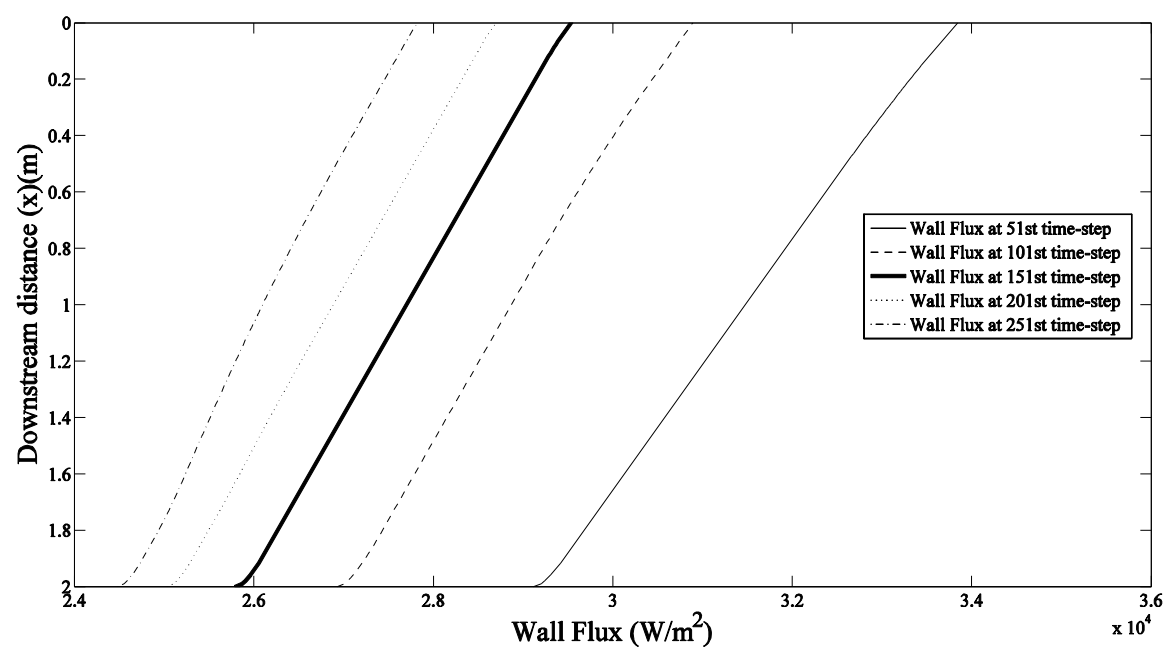

Figure 6.8: Plot of Wall Flux q" $(x, t)$ after every 50 time-steps 


\subsection{Results for "Melting" Duration}

As described earlier in section 5.2 and Appendix B, a shifted time variable namely $\hat{t} \equiv t-\left(t_{M}+\varepsilon\right)$, is used to keep track of the subsequent times $\hat{t}$ of interest. Note that $\hat{t}=0$, is same as $t=\left(t_{M}+\varepsilon\right)$. Extracting the values of the wall temperature $T_{w}\left(x, t_{M}\right)$ and the mean HTF temperature $T_{m}\left(x, t_{M}\right)$ from Fig. 6.6 and Fig. 6.7, the plots are shown again in Fig. 6.9 as function of downstream distance $x$.

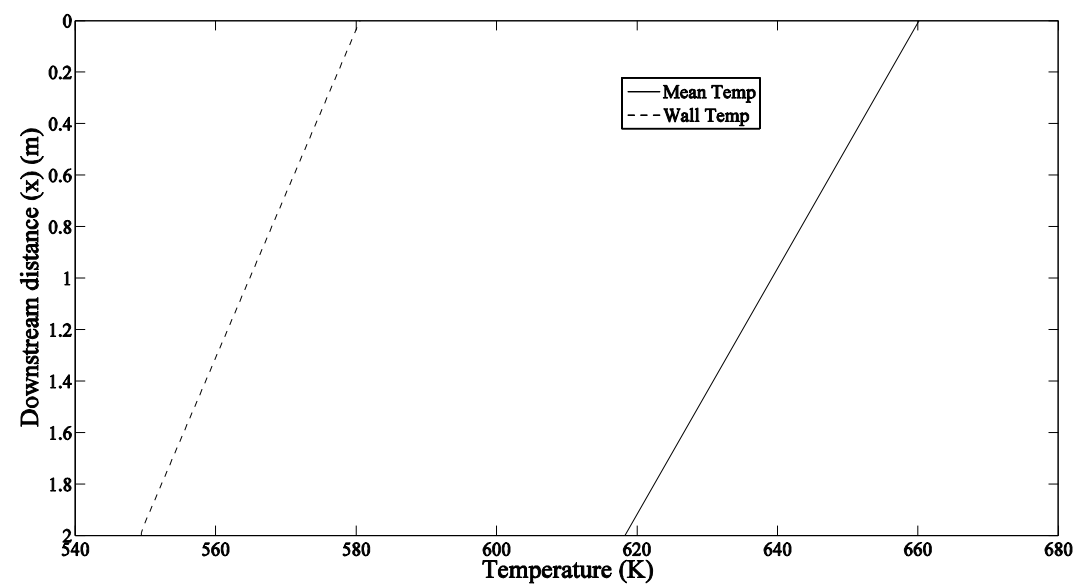

Figure 6.9: Plot of Wall Temperature $T_{w}\left(x, t_{M}\right)$ and Mean Temperature $T_{m}\left(x, t_{M}\right)$

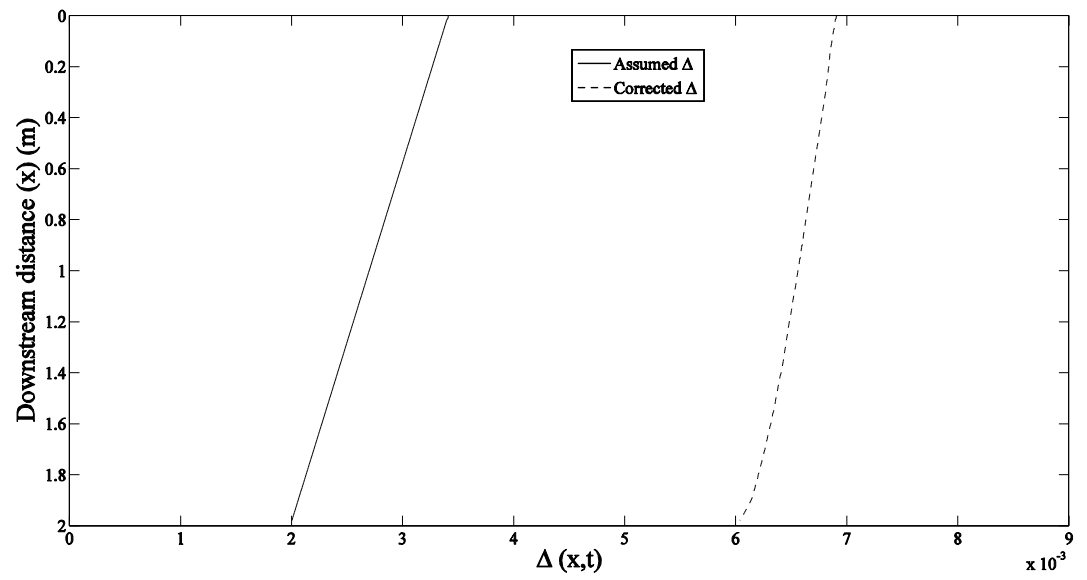

Figure 6.10: Plot of Assumed and Corrected Location of Interface $\Delta(x, \hat{t}=0)$ 
The initial arbitrary choice for the location of the interface $\Delta\left(x, t_{M}+\varepsilon\right)$ at a time $t=t_{M}+\varepsilon$ (see section 5.2 and Appendix B) then corrected using numerical approach described for the "melting" duration (see section 5.2). Plot in Fig. 6.10 show the initially assumed location of the interface $\Delta\left(x, t_{M}+\varepsilon\right)$ and the converged value of the corrected interface $\Delta\left(x, t_{M}+\varepsilon\right)$ location as function of downstream distance.

To capture a gradual evolution of interface $\Delta(x, \hat{t})$, the solution is marched in time-step $\Delta t_{c}$ value of $350 \mathrm{~s}$. For the "melting" duration, Fig. 6.11 shows the converged values of $\Delta(x, t)$ plotted as $\Delta$ versus downstream distance $x$ with time $t\left(\equiv n * \Delta t_{c}\right)$ as a parameter. Figure 6.12 shows temperature within the molten PCM and the solid PCM at $\hat{t}=1400 s$ obtained at $x=\frac{L}{2}$ cross-section of the LHTES system. Figure 6.13 shows the "melting" duration wall temperature $\mathrm{T}_{\mathrm{w}}(\mathrm{x}, \hat{t})$ plotted against downstream distance $x$ with time $\hat{t}$ as a parameter.

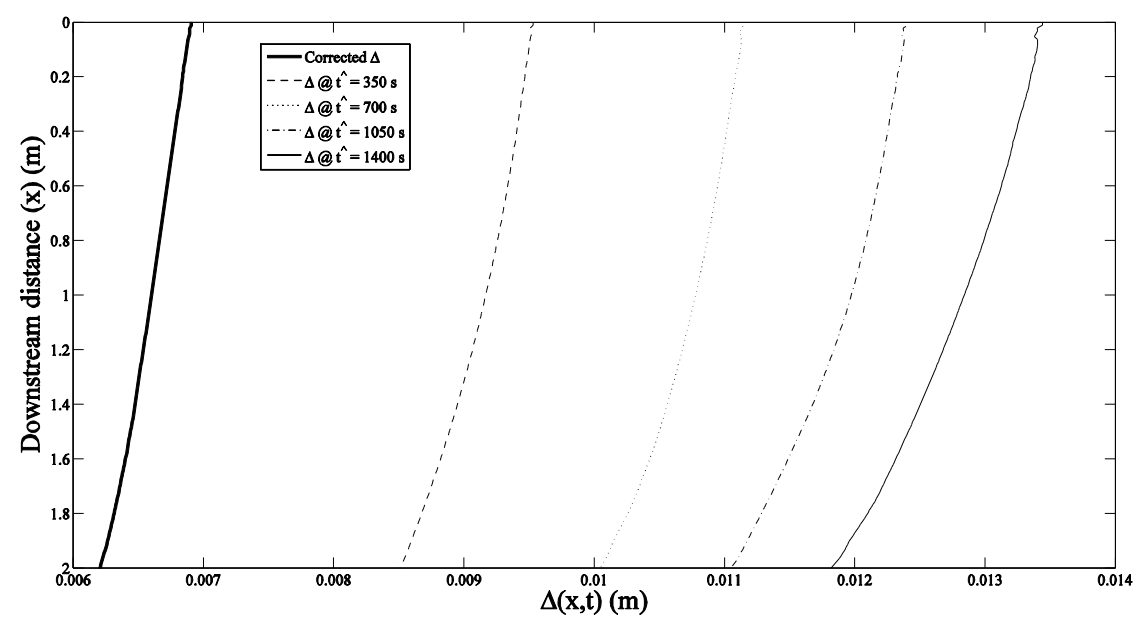

Figure 6.11: Evolution of Interface $\Delta(x, \hat{t})$ 


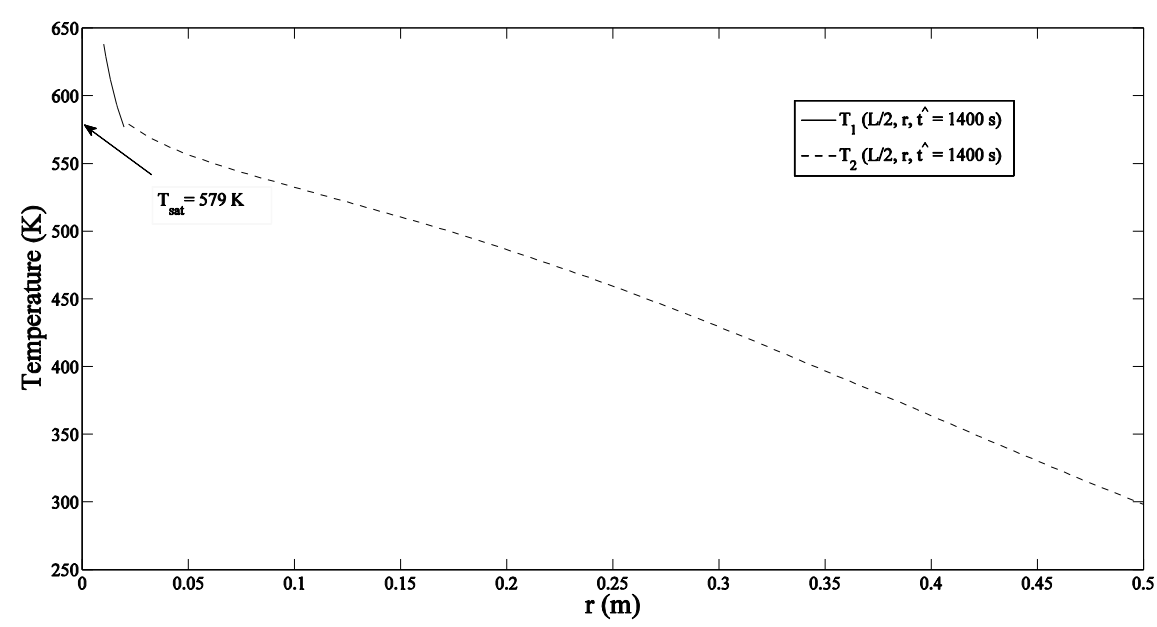

Figure 6.12: Representative plot of the temperature variation in radial direction

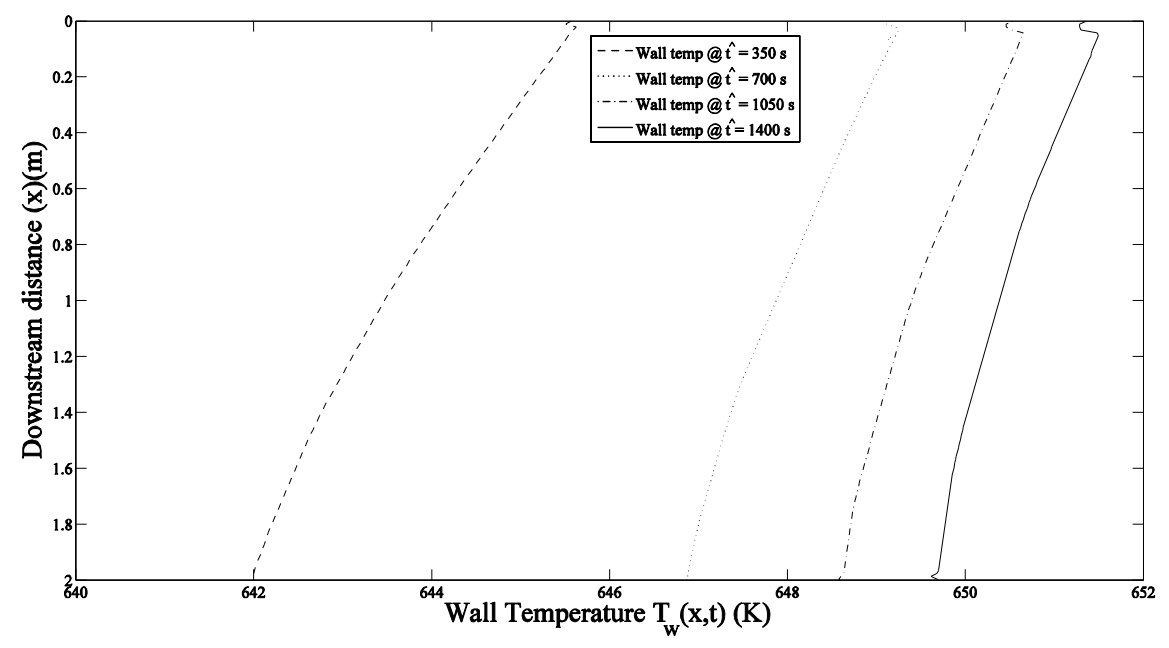

Figure 6.13: Wall Temperature $T_{w}(x, \hat{t}) v / s$ downstream distance ("Melting" duration)

The time-varying natures of the effectiveness $\epsilon_{1}(\hat{t}), \epsilon_{2}(\hat{t})$, and efficiency $\eta(\hat{t})$ (see section 2.2 for definitions) of the LHTES systems as a function of $\hat{t}$ are of interest. The plots for effectiveness $\epsilon_{1}(\hat{t}), \epsilon_{2}(\hat{t})$, and efficiency $\eta(\hat{t})$ with length $L$ as a parameter, are shown in Figs. (6.14)- (6.16). The bigger length of the pipe allows the PCM to extract more heat from the HTF and store it as a latent heat. This is shown in Fig. 6.14, where effectiveness $\epsilon_{1}(\hat{t})$ increases with length of pipe. The part of heat transferred to the PCM from pipe wall; which causes phase change in the PCM, is not dependent on length of the pipe. As shown in Fig. 6.15, the effectiveness $\epsilon_{2}(\hat{t})$ is independent of length of the pipe. 


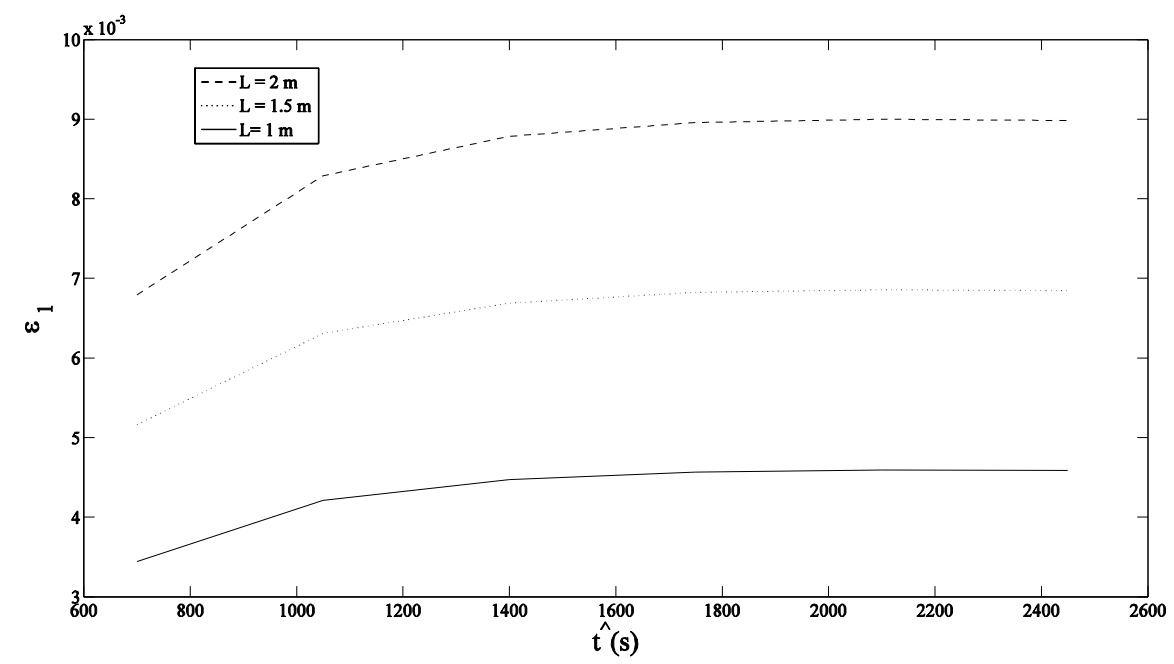

Figure 6.14: Plots of $\epsilon_{1}(\hat{t})$, against time $\hat{t}$ for spatial domains of different lengths

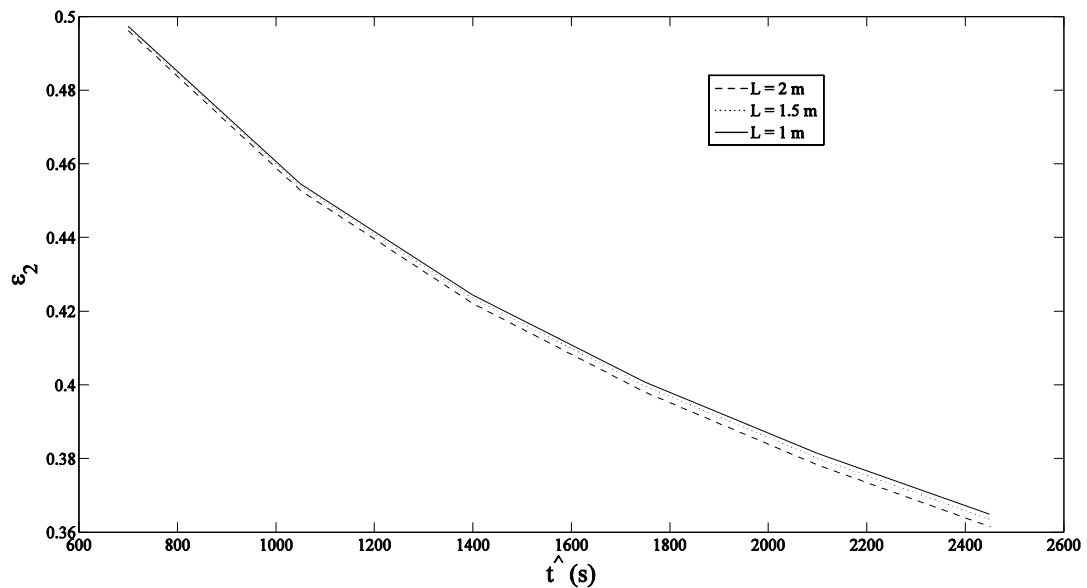

Figure 6.15: Plots of $\epsilon_{2}(\hat{t})$, against time $\hat{t}$ for spatial domains of different lengths

Plots for efficiency $\eta(\hat{t})$ with length $L$ as a parameter are shown in Fig. 6.16. The values of $\eta(\hat{t})$ highlights the importance of the LHTES systems. The pump consumes very small amount of power in circulating the HTF through LHTES system, while PCM stores large amount of thermal energy as a latent heat. As expected, efficiency $\eta(\hat{t})$ decreases if pipe with smaller length is used. 


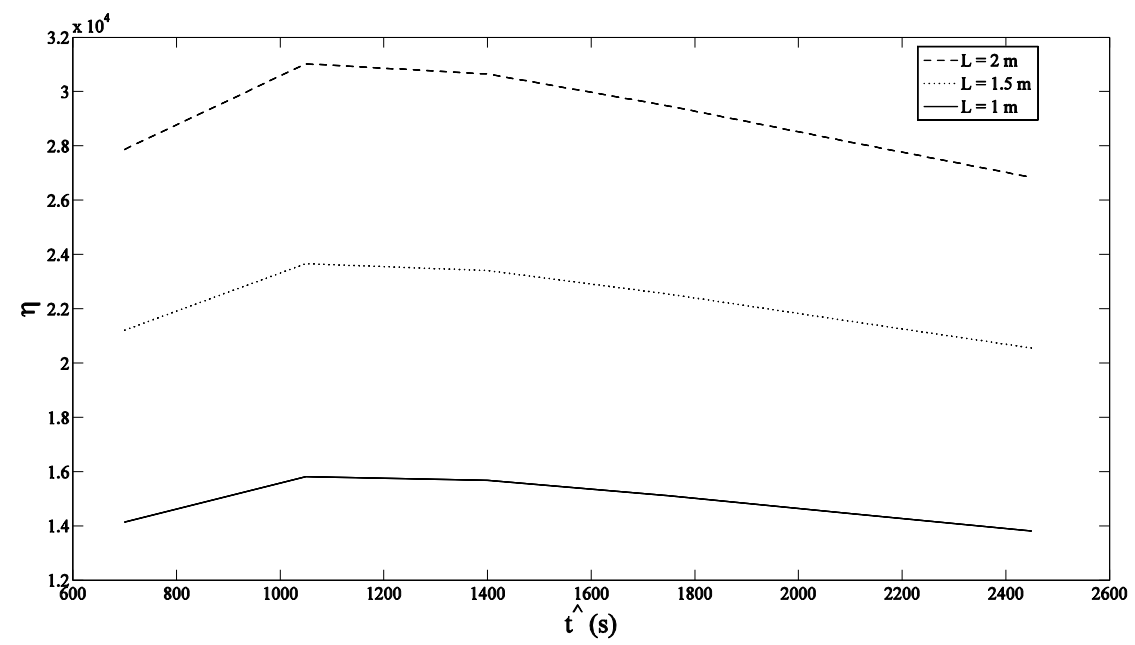

Figure 6.16: Plots of $\eta(\hat{t})$, against time $\hat{t}$ for spatial domains of different lengths

\subsection{Results for "Melting" Duration (Natural Convection within the Molten PCM Modeled Using Ad-hoc model)}

As melt-solid interface evolves in the solid PCM, the buoyancy forces within the molten PCM are increased. Hence, movement of the molten PCM becomes increasingly significant. This movement of the molten PCM increases rate of heat transfer within the molten PCM. The effect of natural convection is estimated using ad-hoc model for thermal conductivity of the molten PCM.

If Rayleigh number $R a_{\Delta}$ for the molten PCM is obtained by following equation:

$$
R a_{\Delta}=\frac{g \beta \overline{\Delta T}(t) * \bar{\Delta}(t)^{3}}{\vartheta_{1} * \alpha_{1}}
$$

where $\overline{\Delta T}(t)=\overline{T_{w}}(x, t)-T_{\text {sat }}$ and $\bar{\Delta}(t)=\frac{1}{L} \int_{0}^{L} \Delta(x, t) * d x$

The Nusselt number correlation (Incropera, 2006) is used to obtain effective thermal conductivity for the molten PCM and it is given by:

$$
N u_{\Delta}(t)=0.42 * R a_{\Delta}{ }^{(1 / 4)} * P r_{1}{ }^{0.012} *\left(\frac{L}{\bar{\Delta}(t)}\right)^{-0.3}
$$

and effective thermal conductivity is obtained by following equation: 


$$
K_{e f f}(t)=K_{1} * N u_{\Delta}(t)
$$

Figure 6.17 shows plot of Rayleigh number $R a_{\Delta}$ versus time in the "melting" duration. As natural convection within the molten PCM leads to better heat transfer rates, the evolution of the interface is faster as compared to the previous case, where natural convection within the molten PCM is neglected. Figure 6.18 shows plots of converged values for interface locations $\Delta(\mathrm{x}, \hat{\mathrm{t}})$ versus downstream distance $x$ plotted after 350 seconds.

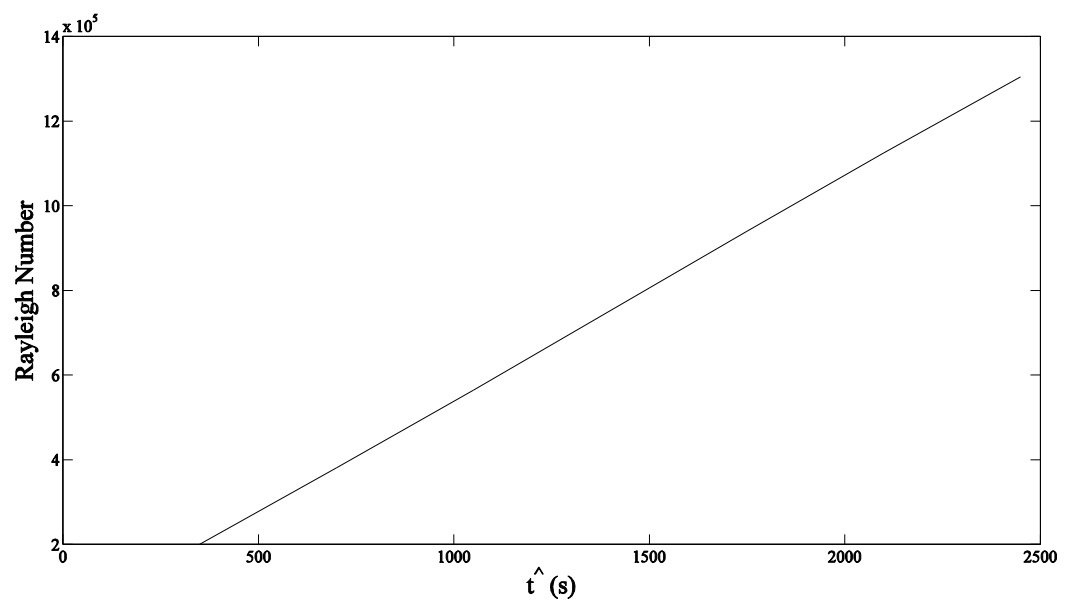

Figure 6.17: Plot of Rayleigh Number $R a_{\Delta}$

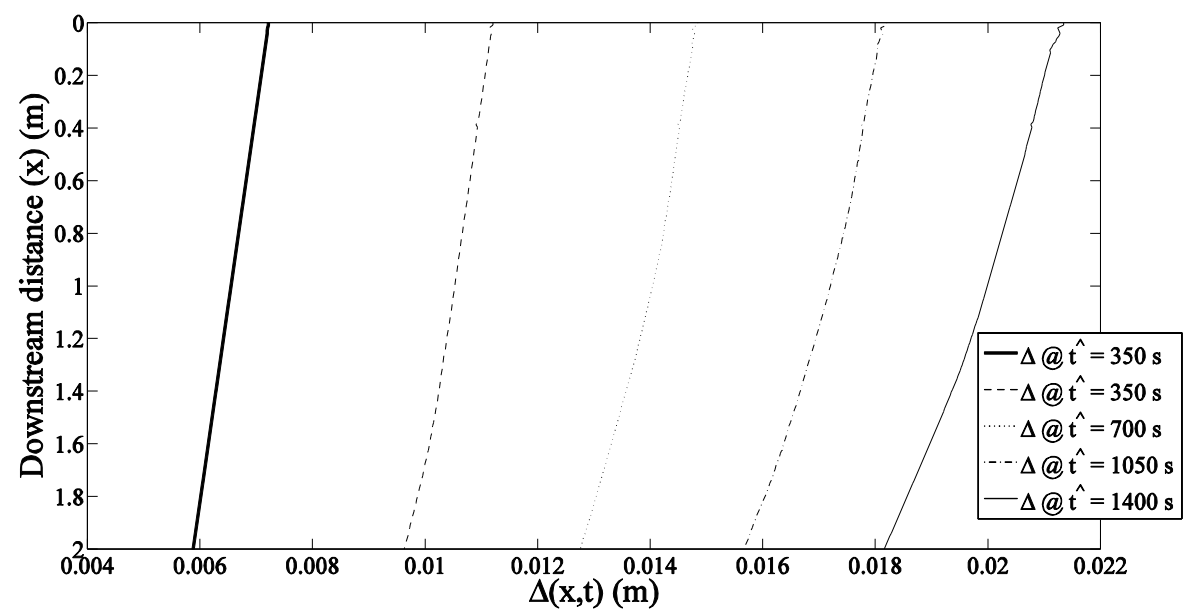

Figure 6.18 : Evolution of Interface $\Delta\left(x, t^{\wedge}\right)$ (with natural convection effects) 
The better heat transfer within the molten PCM (caused by buoyancy forces) leads to faster movement of the melt-solid interface, the effectivity and efficiency of the LHTES system also increases, as shown in Figs. (6.19) - (6.21).

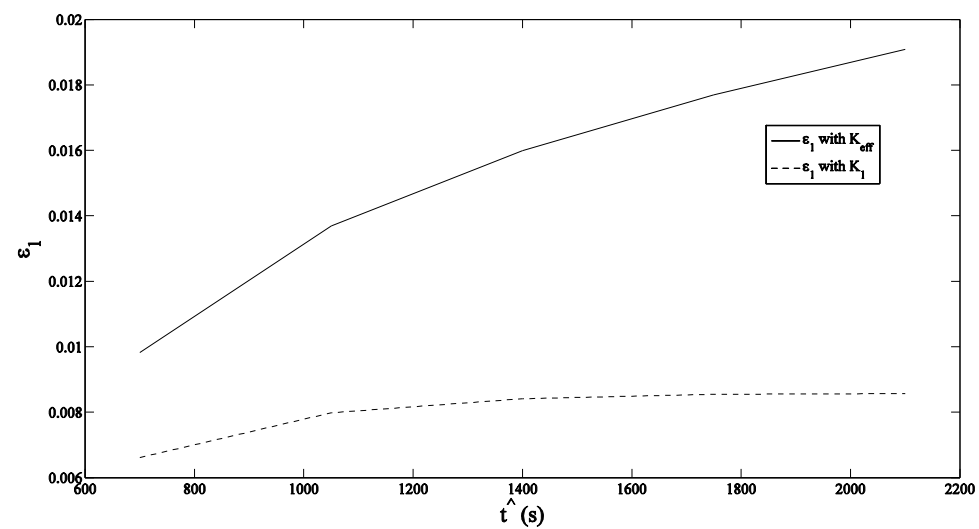

Figure 6.19: Plots of efficiencies $\varepsilon_{1}$ for two different cases

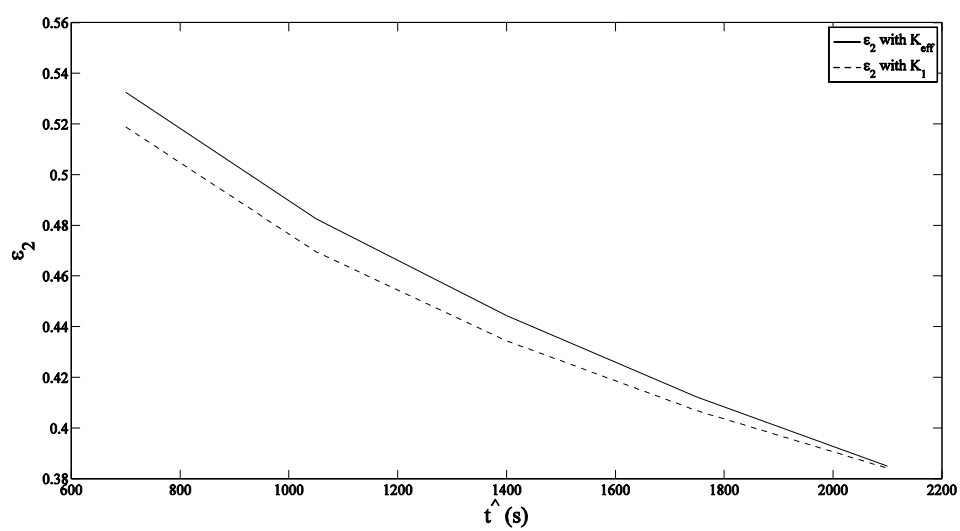

Figure 6.20: Plots of efficiencies $\varepsilon_{2}$ for two different cases

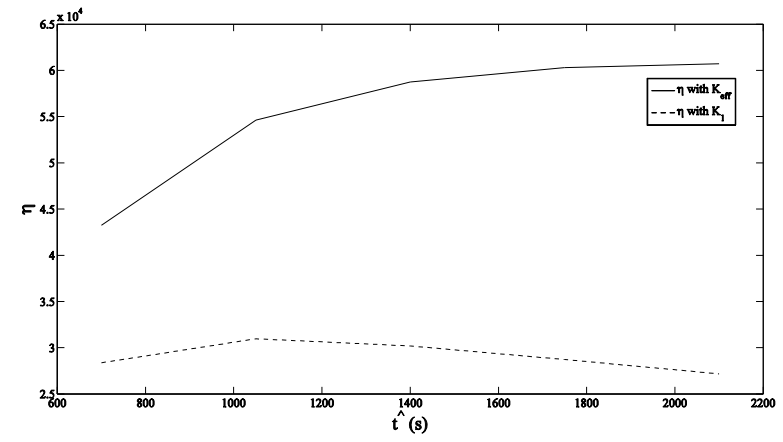

Figure 6.21: Plot of Effectiveness for two different cases 


\subsection{The Comparison with Analytical Solution for Two Phase Stefan Problem:}

It is necessary to validate the results obtained from mathematical modeling and computational simulation tool described here to analytical solution for the Stefan Problem. Neumann similarity solution of the 2-phase Stefan Problem for the interface location is given by:

$$
\bar{\Delta}(t)=2 \lambda \sqrt{\alpha_{1} * t}
$$

where $\lambda$ is obtained by the solution from the following transcendental equation

$$
\frac{S t_{1}}{\exp \left(\lambda^{2}\right) \operatorname{erf}(\lambda)}-\frac{S t_{2}}{v \exp \left(v^{2} \lambda^{2} \operatorname{erfc}(v \lambda)\right.}=\lambda \sqrt{\pi}
$$

Figure 6.22 shows plots for $\bar{\Delta}(t)$ obtained from analytical solution for two phase Stefan problem and $\bar{\Delta}(t)$ obtained from the simulation tool described here. In this case, it is assumed that initially solid PCM is at temperature of $T_{\text {sat }}$ and pipe wall temperature is help at constant temperature higher than $T_{\text {sat }}$ (i.e. $T_{w}(x, t)=T_{\text {sat }}+10$ ). The melt-solid interface evolves by a same distance irrespective of the downstream distance (i.e. $\Delta(x, t)=\bar{\Delta}(t))$. As analytical solution for the Stefan problem neglects superheating of the solid PCM and it assumes insulation condition at outer boundary of the solid PCM, it over predicts the value of the $\bar{\Delta}(t)$.

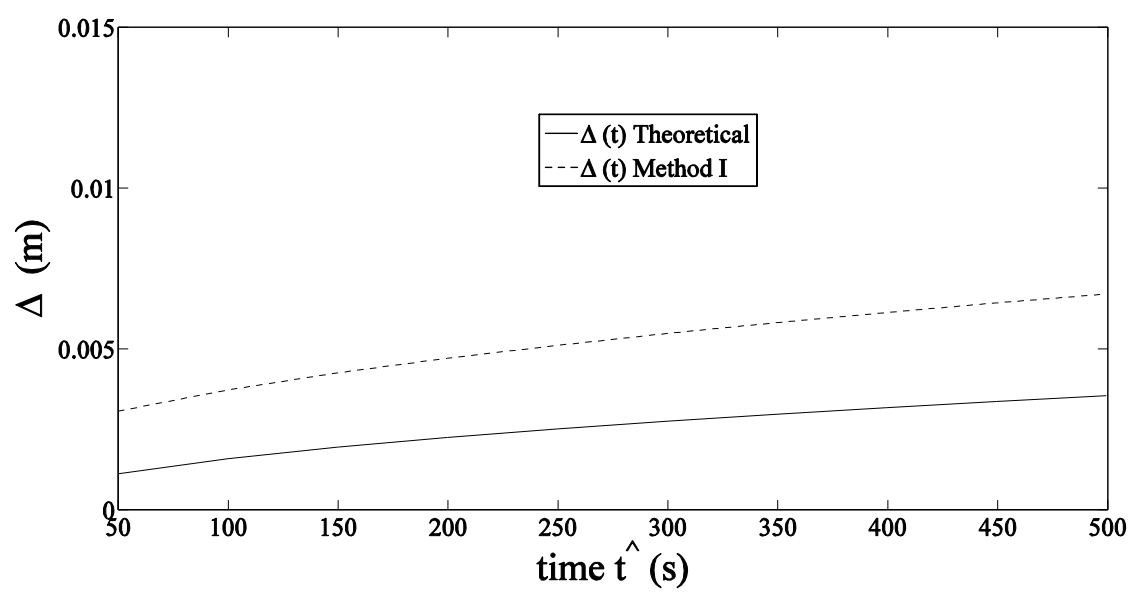

Figure 6.22 : Plots for $\bar{\Delta}(t)$ 


\section{FUTURE RECOMMENDATIONS}

- The mathematical modeling and simulation approach described here successfully captures the physics of transient heat transfer processes occurring during "charging cycle" in LHTES system of interest. This computational tool can be modified (with some minor changes) to model the physics in "discharging cycle" - where PCM solidifies and transfers heat to reversed flow of HTF.

- This computational tool can be modified to account natural convection in the molten PCM and its effects on performance of the LHTES system.

- The effects of other boundary conditions (like insulation boundary condition, convection boundary condition at $r=r_{2}$, etc) can be studied.

- The effects of variables such as inlet temperature $T_{\text {in }}$ and mass flow rate of HTF, thermo- chemical properties of liquid and solid phases of the PCM, etc can be done. Furthermore, the computational tool described here, can be used for parametric study of other important non-dimensional numbers.

- The computational can be used to assess the enhancement of effectiveness and efficient of LHTES, obtained by use of nano-particles and/or heat pipes within the PCM. 


\section{CONCLUSIONS}

The study shows that first principle reliable simulation of the complex LHTES system problem - made of several conjugate problems - is possible. The study presents the first of its kind much needed results on time-varying efficiency and effectiveness of LHTES. The results obtained by this modeling and simulation approach are grid independent. The science behind a reliable prediction of the evolving interface, the role of relative nondimensional numbers, and the interplay of different time-scales have been outlined. The effects of natural convection within the molten PCM on performance of the LHTES system are estimated. This estimate also facilitates a complete CFD modeling of the natural convection effects. Future directions as regard to experimental and modeling works outlined to further advance both the science and application of PCM uses in LHTES system. 


\section{REFERENCES}

Abbott MB, BD. 1989. Computational Fluid Dynamics-An Introduction For Engineers. NY: Longman/Wiley.

Agyenim F, HN. 2010. A review of materials, heat transfer and phase change problem formulation. Renewable and Sustainable Energy Reviews. 14:615-628.

Bauer T, LD. 2009. Sodium Nitrate for High Temperature Latent Heat Storage. International Conference on Thermal Energy Storage- Effstock. :14-17.

C, N. 2007. The Zero-phase Stefan Problem. International Journal of Heat and Mass Transfer. 50:4614-4622.

Castell A, SC. 2008. Natural convection heat transfer coefficients in phase change material (PCM) modules with external vertical fins. Applied Thermal Engineering. 28: $1676-1686$.

Chantasiriwan S, JB. 2009. The Method of Fundamental Solutions for Free Surface Stefan problems. Engineering Analysis with Boundary Elements. 33:529-538.

Delhaye, JM. 1974. Jump Conditions and Entropy Sources in Two Phase. International Journal Multiphase Flow. 1:395-409.

Esen M, AT. 1996. Development of a Model Compatible with Solar Assisted Cylindrical Energy Storage Tank and Variation of Stored Energy with Time for Different Phase Change Materialse For. Energy Conversion Management. 37 (12):1775-1785.

Incropera DB. 2006. Fundamentals of Heat and Mass Transfer. Sixth ed. :Wiley.

J, M. 2004. Numerical Simulation of Melting in Two-dimensional. Journal of Computational Physics. 198:243-264.

Kenisarin M, MK. 2007. Solar energy storage using phase change materials. Renewable and Sustainable Energy Reviews. 11:1913-1965.

Michels H, PP. R. 2007. Cascaded Latent Heat Storage for Parabolic Trough Solar Power Plants. Solar Energy. 81:829-837.

Mitra S, NA. 2011. A Quasi One-dimensional Method and Results for Steady Annular/Stratified Shear and Gravity Driven Condensing Flows. International Journal of Heat and Mass Transfer. 54:3761-3776. 
Narain A, LQ. 2004. Direct Computational Simulations for Internal Condensing Flows and Results on Attainability/Stability of Steady Solutions, Their Intrinsic Waviness, and Their Noise Sensitivity. Jornal of Applied Mechanics. 71:69-88.

Onyejekwe OO, OO. 2011. Numerical Solutions of the One-phase Classical Stefan Problem. Advances in Engineering Software. 42:743-749.

Peng Q, DJ. 2010. The preparation and properties of multi-component molten salts. Applied Energy. 87:2812-2817.

Robak C, BT. 2011. Enhancement of Latent Heat Energy Storage Using Embedded Heat Pipes. International Journal of Heat and Mass Transfer. 54:3476-3484.

Sharma A, TV. 2009. Review on thermal energy storage with phase change. Renewable and Sustainable Energy Reviews. 13:318-345.

Shin D, BD. 2010. Enhanced Thermal Properties of PCM Based Nanofluid for Solar Thermal Energy Storage. International Conference on Energy Sustainability. ES2010-90293.

Shin D, BD. 2010. Enhancement of specific heat capacity of high-temperature silicananofluids synthesized in alkali chloride salt eutectics for solar thermal-energy. International Journal of Heat and Mass Transfer. 54:1064-1070.

Shin D, BD. 2009. Investigation of Nanofluids for Solar Thermal Storage. International Conference on Energy Sustainability. ES2009-90465.

Shmueli H, ZG. 2010. Melting in a Vvertical Cylindrical Tube: Numerical Iinvestigation and Comparison. International Journal of Heat and Mass Transfer. 53:4082-4091.

T, C. 2000. Numerical Analysis of Nonlinear Multiphase Stefan. Computers and Structures. 75:225-233.

Tzai-Fu, C. (2000). Numerical Analysis of Nonlinear Multiphase Stefan Problems. Computers and Structures. 75:225-233.

Wang S., FA. 2010. A comprehensive Numerical Model for Melting with Natural Convection. International Journal of Heat and Mass Transfer. 53:1986-2000

Weng Y, CH. 2011. Heat Pipe with PCM for Electronic Cooling. Applied Energy. 88: $1825-1833$.

Ye W, ZD. 2011. Numerical Simulation on Phase-change Thermal Storage/release in a Plate-fin Unit. Applied Thermal Engineering. 31:3871-3884. 
Zalba B, MJ. 2003. Review on thermal energy storage with phase. Applied Thermal Engineering. 23:251-283. 


\section{Appendix A. Various Time-scales of Interest}

The LHTES system has several conjugate unsteady heat transfer problems over "before melting" and "melting" duration. Each unsteady problem has its own characteristic time. For example: time scale associated with transient and forced convection of heat in the HTF flow, transient conduction of heat in the solid PCM, transient conduction of heat in the molten PCM, time scales associated with the evolution of the interest, etc. Evaluation of these characteristic times enables one to select the time-steps $\Delta t_{c}$ that are best for the overall simulation of the LHTES system over "before melting" and "melting" durations. An analysis and investigation of these characteristic times reveal that the slow (or "rate limiting") time scale which control the LHTES operations are transient conduction in the solid PCM and time scale associated with the movement of the interface.

\section{Characteristic Times Associated with Condition in the Solid and Molten PCM}

\section{Solid PCM:}

The conduction equation (12) in section 4.3, under non-dimensionalization of:

$$
\left\{T_{2}, t, t\right\} \equiv\left\{\left(T_{\text {in }}+\Delta t\right) \theta_{2}, \quad\left(L_{2}\right) \hat{r}_{2}, \quad \Delta t_{c_{2}} \tau_{2}\right\}
$$

where $\Delta t \equiv T_{\text {in }}-T_{\infty}, L_{2} \equiv D_{2}-D_{1}$, and $\Delta t_{c_{2}} \equiv \frac{L_{2}^{2}}{\alpha_{2}}$, leads to its well known nondimensional version:

$$
\frac{\partial \theta_{2}}{\partial \tau_{2}} \equiv \nabla^{2} \theta_{2}
$$

The non dimensional time $\Delta t_{c_{2}} \equiv \frac{L_{2}{ }^{2}}{\alpha_{2}}$ is the characteristic time for the heat conduction through the solid PCM.

\section{Molten PCM:}


If one ignores natural convection, the unsteady conduction equation under nondimensionalization:

$$
\left\{T_{1}, r, t\right\} \equiv\left\{\left(T_{\text {in }}+\Delta t\right) \theta_{1}, \quad\left(\Delta_{m}(t)\right) \hat{r}_{1}, \quad \Delta t_{c_{1}} \tau_{1}\right\}
$$

where $\Delta t \equiv T_{\text {in }}-T_{\infty}, \Delta_{m}(t)$ is the representative mean thickness of the melt at $t>t_{M}$, and $\Delta t_{c_{1}} \equiv \frac{\Delta_{m}(t)^{2}}{\alpha_{2}}$, leads to its well known non-dimensional version:

$$
\frac{\partial \theta_{1}}{\partial \tau_{2}} \equiv \nabla^{2} \theta_{1}
$$

The non dimensional time $\Delta t_{c_{1}} \equiv \frac{\Delta_{m}(t)^{2}}{\alpha_{2}}$ is the characteristic time for the heat conduction through the molten PCM.

\section{Characteristic Time Associated with Transient and Force Convection of Heat on HTF Flow}

For forced convection of heat in the HTF flow, the differential form for energy balance is expressed as

$$
\rho_{F} C_{P_{F}}\left[U_{r} \frac{\partial T_{F}}{\partial r}+U_{z} \frac{\partial T_{F}}{\partial z}+\frac{\partial T_{F}}{\partial t}\right]=k_{t_{F}} \nabla^{2} T_{F}
$$

where $U_{r}, U_{z}, T_{F}$ respectively represent the radial component of velocity, axial component of velocity, and the local temperature of the fluid (of density $\rho_{F}$, specific heat $C_{P_{F}}$, and turbulent conductivity $k_{t_{F}}$ ). The boundary conditions that affect the temperature variations governed by equation (A-5), is the time-variations of the wall temperature $T_{w}(x, t)$. The wall temperature variations, in turn, is governed by solid PCM characteristic time $\Delta t_{c_{2}}$ over the "before melting" duration and the melt-interface evolution time $\Delta t_{M-I}$ (to be defined) over the "melting" duration.

The forced convection characteristic time $\Delta t_{F-f c}$ (due to the first two terms on the left side of equation (A-5)) for "before melting" duration is estimated from heat-exchange 
energy balance across the pipe-wall over a period of time. This is estimated through following energy balance at the pipe-wall:

$$
\begin{gathered}
\left(\frac{\dot{M} C_{P_{F}} \Delta T_{m}{ }^{o}}{\Delta t_{c_{F}}}\right) \Delta t_{F-f c}=O\left[k_{2} * \frac{\partial T_{2}}{\partial r} * \pi D_{1} L\right] \Delta t_{c_{2}} \\
=k_{2} * \frac{\Delta T_{m}{ }^{o}}{L_{c_{2}}} * \pi D_{1} L * \Delta t_{c_{2}}
\end{gathered}
$$

In equation (A-6), $\Delta T_{m}{ }^{o} \equiv T_{i n}-T_{\infty}$ is a characteristic temperature-difference. Thus, for "before melting" one obtains:

$$
\frac{\Delta t_{F-f c}}{\Delta t_{c_{2}}} \equiv \frac{k_{2} * \pi D_{1} L}{D_{2}-D_{1}} * \frac{1}{\rho_{F} C_{P_{F}} Q_{F}}
$$

$$
\equiv \ll 1
$$

Thus the time $\Delta t_{F-f c}$ for the HTF flow to adjust to changes in wall temperature is very rapid and the flow effectively adjusts to changes in wall temperature. Also, for the "before melting" duration, the effect arising from the $\frac{\partial T_{F}}{\partial t}$ term in equation (A-5) is also governed by changes in wall temperature $T_{w}(x, t)$, which according to equation (A-2), is governed by $\Delta t_{c_{2}}$. Therefore,

$$
\begin{gathered}
O\left[\frac{\partial T_{F}}{\partial t}\right]=\frac{\Delta T_{m}{ }^{o}}{\Delta t_{c_{2}}} * O\left[\frac{\partial \theta_{2}}{\partial \tau_{2}}\right] \\
\cong \frac{\Delta T_{m}^{o}}{\Delta t_{c_{2}}} * 1
\end{gathered}
$$

The estimate from equation (A-8) can be substituted in equation (A-5), to assess the size of the transient conduction terms with respect to the forced convection terms, which sizes are of $\frac{U}{L} \Delta T_{m}{ }^{o}$. 
It is easily verified that

$$
\frac{U}{L} \gg \frac{1}{\Delta t_{c_{2}}}
$$

Because on multiplication with $\frac{L D_{1}}{\vartheta_{F}}$, one gets:

$$
\frac{L D_{1}}{\vartheta_{F}} \equiv R e_{D} \gg \frac{L D_{1}}{\vartheta_{F}} * \frac{\alpha_{2}}{L_{C}^{2}}
$$

Equations (A-8)-(A-10) confirm the obvious result that transient heat convection results are negligible compared to forced convection. Because of equation (A-7), one concludes that of time-steps $\Delta t_{c}$ for transient conduction in the solid PCM for the "before melting" duration is set to:

$$
\Delta t_{c} \equiv 0.025 * \Delta t_{c_{2}}
$$

Then, one continues to have, $\Delta t_{c} \gg \Delta t_{F-f c}$. Therefore, one can continue to use the quasisteady forced convection analysis of section 4.2 .

\section{Time-scale $\Delta t_{M-I}$ associated with the evolution of the interface}

The interface evolution equation in Appendix $\mathrm{C}$ yields:

$$
\begin{array}{cc}
O\left[\frac{\partial \Delta}{\partial t}\right]=O[\bar{V}] \\
\text { or } \quad \frac{\Delta_{m}(t)}{\Delta t_{M-I}} \cong \frac{k_{1}\left(T_{i n}-T_{s a t}\right)}{\rho_{F} * h_{s f} \Delta_{m}(t)}
\end{array}
$$

The same order of magnitude estimate is also obtained from:

$$
\dot{m}_{m e l t} * h_{s f}=k_{1} \frac{\left(T_{i n}-T_{s a t}\right)}{\Delta_{m}(t)} \pi D_{1} L
$$

where $\dot{m}_{m e l t}$ is approximated as $\dot{m}_{m e l t} \cong \frac{\rho_{1} \pi D_{1} L * \Delta_{m}(t)}{k_{1} *\left(T_{\text {in }}-T_{\text {sat }}\right)}$. 
Thus, one obtains:

$$
\begin{array}{r}
\Delta t_{M-I} \equiv \frac{\rho_{1 * \Delta_{m}}(t)^{2} * h_{s f}}{k_{1} * J a} \\
=\frac{\Delta t_{M-\text { cond }}}{J a}
\end{array}
$$

where $J a \equiv \frac{C_{P_{1}} *\left(T_{i n}-T_{s a t}\right)}{h_{s f}} \ll 1$

A concurrent look at the times $\Delta t_{c_{2}}$ and $\Delta t_{M-I}$ for times $t \gg t_{M}$ helps one to decide on the apparent time-scale " $\Delta t_{c}$ " for the "melting" duration. By this procedure, for the results reported in Figs $6.4-6.9$, it was found that $\left.\left.\Delta t_{c}\right|_{\text {melting }} \cong 0.5 * \Delta t_{c}\right|_{\text {before-melting }}$ was most appropriate for gradual evolution of the interface over duration of interest. 


\section{Appendix B. Rapid Encapsulation of the HTF pipe by PCM Melt}

At $t=t_{M}$, melting of the solid PCM begins at the inlet section of the pipe surface (see point $\mathrm{M}$ in Fig. 2.2). At $t=t_{M}$, (the end of "before melting" duration), the mean HTF temperature $T_{m}\left(x, t_{M}\right)$ is well above $T_{s a t}$ at all $x$ locations and this causes wall temperature $T_{w}(x, t)$ to exceed $T_{\text {sat }}$ very rapidly over $t_{M} \leq t \leq t_{M}+\varepsilon$. As a result, the melt-front point in Fig. 2.2 unfolds very rapidly to yield a thin interface surrounding the pipe from $x=0$ to $x=L$.

\section{Analysis for an estimate for the time duration " $\varepsilon$ ":}

The melting begins at time $t=t_{M}$. Assume that at $t=t_{M}+\varepsilon$, the interface location at $x=0$ is arbitrarily modeled as a thin straight line encapsulating the pipe wall and is given by:

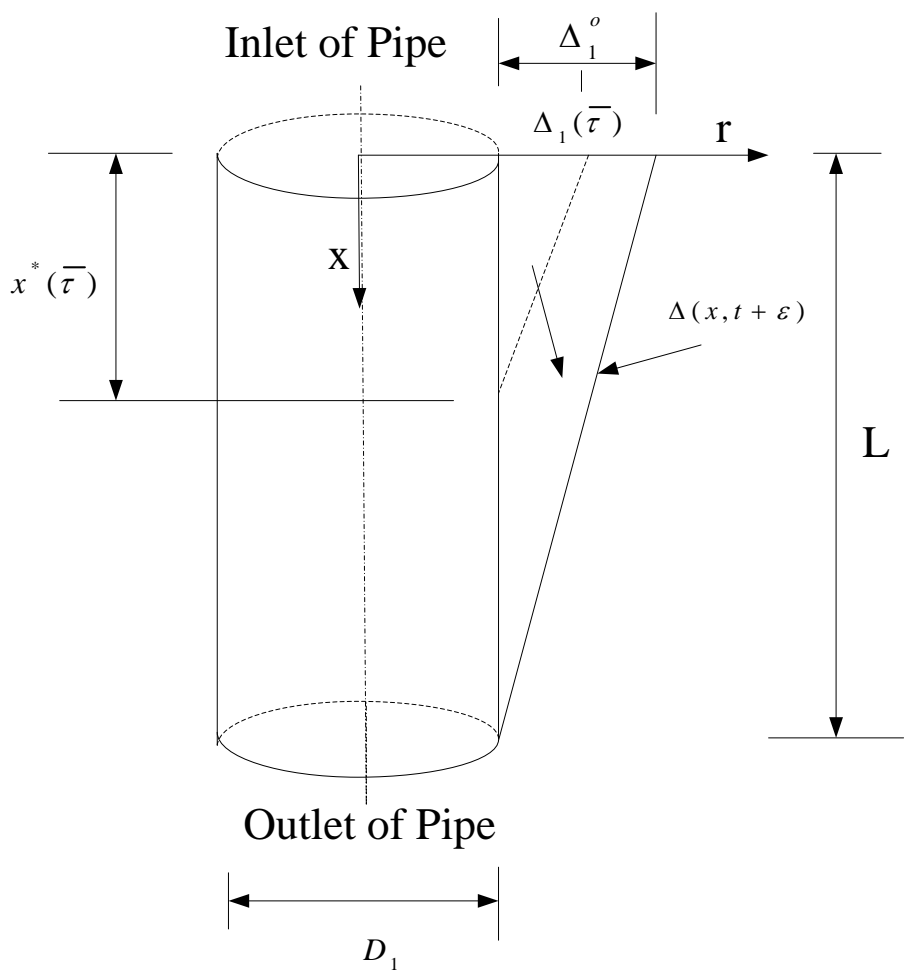

Figure .1: Schematic Representative for Movement of $x^{*}$ 


$$
\Delta\left(x, t_{M}+\varepsilon\right)=\Delta_{1}^{o}-\frac{\Delta_{1}^{o}}{L} x, \text { for } 0 \leq x \leq L
$$

where $\Delta_{1}^{o} \equiv \Delta\left(0, t_{M}+\varepsilon\right)$. Furthermore, in order to get an estimate of the length at time duration $\varepsilon$, it is assumed that the melt-front locations for $t_{M} \leq \bar{\tau} \leq t_{M}+\varepsilon$ is a series of paraller straight lines (see Fig. B.1) given by:

$$
\Delta(x, \bar{\tau})=\Delta_{1}(\bar{\tau})-\frac{\Delta_{1}(\bar{\tau})}{x^{*}(\bar{\tau})} * x, \text { for } 0 \leq x \leq L
$$

where $\frac{\Delta_{1}(\bar{\tau})}{x^{*}(\bar{\tau})}=\frac{\Delta_{1}{ }^{o}}{L}$

The rapid movement of the solid and liquid PCM's points on the surface of the pipe is to be captured by the movement of the triple-point, given by $x=x^{*}(\bar{\tau})$ with $x^{*}\left(t_{M}\right)=0$ and $x^{*}\left(t_{M}+\varepsilon\right)=L$. Note that, at any intermediate instant $\bar{\tau}$, the mean thickness $\Delta_{m}(\bar{\tau})$ is:

$$
\begin{aligned}
\Delta_{m}(\bar{\tau})= & \frac{1}{x^{*}} \int_{0}^{x^{*}}\left\{\frac{\Delta_{1}^{o}}{L} * x^{*}(\bar{\tau})-\frac{\Delta_{1}^{o}}{L} * x\right\} d x \\
& =\frac{\Delta_{1}^{o}}{L} * \frac{x^{*}(\bar{\tau})}{2}
\end{aligned}
$$

Furthermore, at any intermediate instant $\bar{\tau}$, the rate of heat supplied by the HTF fluid to the thin molten PCM over $0 \leq x \leq x^{*}(\bar{\tau})$ is approximately equal to the rate pf latent heat absorbed by the melting PCM. This means:

$$
\dot{M}_{F} * C_{P_{F}} *\left(T_{\text {in }}-T_{\text {sat }}\right) \cong \frac{d}{d \bar{\tau}}\left[m_{m e l t}(\bar{\tau}) * h_{s f}\right]
$$

where $m_{\text {melt }}(\bar{\tau}) \equiv \rho_{1} * \frac{1}{2} \Delta_{1}(\bar{\tau}) x^{*}(\bar{\tau}) * \pi D_{1}=\frac{1}{2} \rho_{1} * \frac{\Delta_{1}{ }^{o}}{L} x^{*}(\bar{\tau})^{2} \pi D_{1}$.

Equation (B-4) implies

$$
\frac{d}{d \bar{\tau}}\left[\frac{1}{2} \rho_{1} * \frac{\Delta_{1}^{o}}{L} x^{*}(\bar{\tau})^{2} * h_{s f} \pi D_{1}\right]=\dot{M}_{F} * C_{P_{F}} *\left(T_{\text {in }}-T_{\text {sat }}\right)
$$




$$
\text { or, } \begin{aligned}
\frac{d}{d \bar{\tau}}\left[\frac{1}{2} \rho_{1} * \frac{\Delta_{1}^{o}}{L} x^{*}(\bar{\tau})^{2} * h_{s f} \pi D_{1}\right] & =\frac{\dot{M}_{F} * C_{P_{F}} *\left(T_{\text {in }}-T_{s a t}\right) 2 L}{\rho_{1} * \Delta_{1}{ }^{o} * h_{s f} * \pi D_{1}} \\
& \equiv C
\end{aligned}
$$

Integration of (B-5), from $\bar{\tau}=t_{M}$ where $x^{*}\left(t_{M}\right)=0$ to $\bar{\tau}=t_{M}+\varepsilon$ where $x^{*}\left(t_{M}+\varepsilon\right)=$ $L$, yields:

$$
\begin{aligned}
& \qquad L^{2}=C * \varepsilon \\
& \qquad \begin{aligned}
\varepsilon & =\frac{L^{2}}{C} \\
& =\frac{\rho_{1} * \Delta_{1}^{o} * h_{s f} * \pi D_{1} L}{\dot{M}_{F} * C_{P F} *\left(T_{\text {in }}-T_{s a t}\right) 2}
\end{aligned}
\end{aligned}
$$

If, in equation (C-7), one uses the estimate of $\Delta_{1}{ }^{o}$ obtained from the energy balance estimate for time $t=t_{M}+\varepsilon$ given by:

$$
\frac{k_{1}\left(T_{\text {in }}-T_{\text {sat }}\right)}{\Delta_{1}^{o} / 2} \pi D_{1} L \cong \dot{M}_{F} * C_{P} *\left(T_{\text {in }}-T_{\text {sat }}\right)
$$

One obtains:

$$
\varepsilon=\frac{\rho_{1} * h_{s f} * k_{1} *\left(\pi D_{1} L\right)^{2}}{\left\{\dot{M}_{F} * C_{P_{F}}\right\}^{2}\left(T_{\text {in }}-T_{s a t}\right)}
$$

The estimate in equation (C-9) implies that, the encapsulation time $\varepsilon$ is indeed numerically very small. For example for the problem discussed here, one obtains:

$$
\begin{aligned}
O(\varepsilon) \cong O(5.71 & \left.* 10^{-4}\right) s \ll \Delta t_{c} \\
& \cong\left\{\begin{array}{c}
700 s \text { (for "before melting" duration) } \\
350 \mathrm{~s} \text { (for melting" duration) }
\end{array}\right.
\end{aligned}
$$

Since, the value of $\varepsilon$ is very small, compared to LHTES times of interest, it is not worthwhile to understand the complex physics of melting over $t_{M} \leq t \leq t_{M}+\varepsilon$. In fact, starting from arbitrary estimate of $\Delta\left(x, t_{M}+\varepsilon\right)$ in equation (B-1), one can numerically solve the problem for larger times $t \gg t_{M}+\varepsilon$. Then, by backward exploration from times at which interface locations are not affected by the arbitrary choice of the assumed interface location, one can improve arbitrary guess for $\Delta\left(x, t_{M}+\varepsilon\right)$. The improvement on guessed location of the interface is implemented iteratively. This iterative correction will 
lead to converged $\Delta\left(x, t_{M}+\varepsilon\right)$ for which the forward marching $\left(t>t_{M}+\varepsilon\right)$ unsteady solutions for $\Delta(x, t)$ are no longer affected by further improvements on the initial interface location profile $\Delta\left(x, t_{M}+\varepsilon\right)$. 


\section{Appendix C. Equations Modeling the Physics -based Requirements that must be met at Solid-Liquid Interface}

Governing equations (see (Delhaye 1974; Narain 2004)) obtained from the requirements of kinematics, thermodynamics, balance laws (mass and energy), and continuity of tangential velocities are discussed in this section.

Let any vector $\overrightarrow{\boldsymbol{V}}$ in cylindrical coordinates be represented as: $\overrightarrow{\boldsymbol{V}}=V_{r} \widehat{\boldsymbol{e}_{\boldsymbol{r}}}+V_{\theta} \widehat{\boldsymbol{e}_{\boldsymbol{\theta}}}+$ $V_{x} \widehat{\boldsymbol{e}_{\boldsymbol{x}}}$, where $\widehat{\boldsymbol{e}_{\boldsymbol{r}}}, \widehat{\boldsymbol{e}_{\boldsymbol{\theta}}}$, and $\widehat{\boldsymbol{e}_{\boldsymbol{x}}}$ are unit vectors along $r, \theta$, and $x$ direction. Let the solidliquid interface be represented by: $r=r_{1}+\Delta(x, t)$. Alternatively, the interface between molten PCM and solid PCM can be implicitly represented as:

$$
\emptyset(r, \theta, x, t)=r-r_{1}-\Delta(x, t)=0
$$

Where, by axi-symmetry, $\varnothing$ is independent of $\theta$. Furthermore, from and henceforth, the values of interior variables at the interface are denoted by a superscript ' $i$ '. The unit normal at any point on the interface, directed from the molten liquid PCM towards the

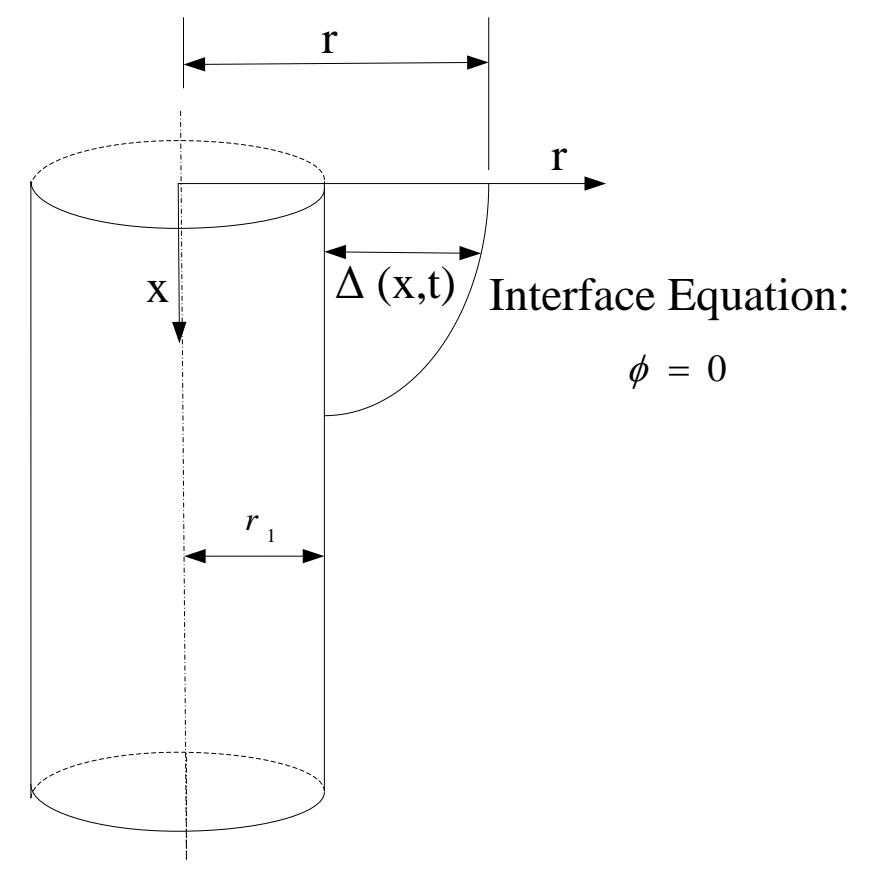

Figure A.1: Cylindrical Co-ordinates System 
solid PCM, is denoted by $\widehat{\boldsymbol{n}}$ and is equal to $\boldsymbol{\nabla} \emptyset /|\nabla \emptyset|$. The unit tangent at any point on the interface, directed towards increasing $\mathrm{x}$, is denoted by $\hat{\boldsymbol{t}}$. The molten phase is modeled as stationary liquid here. For a more realistic natural convection modeling, it could also be modeled as a Newtonian fluid with non-constant for temperature dependent density, under Boussienesq approximation (Incropera 2006).

- The surface velocity $\boldsymbol{v}_{\boldsymbol{s}}$ of a point on the interface $(\varnothing=0)$ at time $t$ is associated with this point's movement to a new mapped position on the interface at time $t+\Delta t_{c}$. All such mappings must be such that the normal component of this $\boldsymbol{v}_{\boldsymbol{s}}$ is given by:

$$
\boldsymbol{v}_{\boldsymbol{s}} \cdot \widehat{\boldsymbol{n}}=-\left(\frac{\partial \emptyset}{\partial t}\right) /|\nabla \emptyset|
$$

Noting that, in cylindrical co-ordinates, we have: $\boldsymbol{\nabla} \emptyset=-\frac{\partial \Delta}{\partial x} \widehat{\boldsymbol{e}_{\boldsymbol{x}}}+(1) \widehat{\boldsymbol{e}_{\boldsymbol{r}}} \boldsymbol{\nabla} \emptyset$, it follows

$$
\begin{aligned}
& \text { that }|\boldsymbol{\nabla} \emptyset|=\sqrt{1+\left(\frac{\partial \Delta}{\partial x}\right)^{2}}=\sqrt{1+\Delta_{x}^{2}} \text {. Therefore, } \\
& \qquad \begin{array}{c}
\widehat{\boldsymbol{n}}=\frac{\boldsymbol{\nabla} \emptyset}{|\nabla \emptyset|}=\frac{-\frac{\partial \Delta}{\partial x} \widehat{\boldsymbol{e}_{\boldsymbol{x}}}+(1) \widehat{\boldsymbol{e}_{\boldsymbol{r}}}}{\sqrt{1+\Delta_{x}^{2}}}=\frac{-\frac{\partial \Delta}{\partial x}}{\sqrt{1+\Delta_{x}^{2}}} \widehat{\boldsymbol{e}_{x}}+\frac{(1)}{\sqrt{1+\Delta_{x}^{2}}} \widehat{\boldsymbol{e}_{\boldsymbol{r}}} \\
\text { and } \frac{\partial T}{\partial n}=\boldsymbol{\nabla} T \cdot \widehat{\boldsymbol{n}}=\left[\frac{\partial T}{\partial x} \widehat{\boldsymbol{e}_{\boldsymbol{x}}}+\frac{\partial T}{\partial r} \widehat{\boldsymbol{e}_{\boldsymbol{r}}}\right] \cdot\left[\frac{-\frac{\partial \Delta}{\partial x}}{\sqrt{1+\Delta_{x}^{2}}} \widehat{\boldsymbol{e}_{\boldsymbol{x}}}+\right. \\
11+\Delta x 2 \boldsymbol{e r}
\end{array}
\end{aligned}
$$

$$
=\frac{\frac{\partial T}{\partial r}-\Delta_{x} \cdot \frac{\partial T}{\partial x}}{\sqrt{1+\Delta_{x}{ }^{2}}}
$$

The expression above for $\frac{\partial T}{\partial n}$ gives variation of temperature along the normal direction from the interface.

- The solid PCM is stationary; therefore, the absolute velocity $\overrightarrow{\boldsymbol{V}_{2}}$ in the PCM is zero everywhere. Since, the tangential component of the molten and solid (which is stationary) PCM velocities at the interface must be continuous, it follows that:

$$
v_{1}^{i} \cdot \hat{t}=v_{2}^{i} \cdot \hat{t}=0
$$


- The interface (interface mass transfer rates per unit area per unit time) mass-fluxes $\dot{m}_{S K}$ and $\dot{m}_{L K}$ are also determined by the kinematic restrictions imposed on the normal components of interfacial values of the phase velocities (for the molten and solid phases of the PCM) relative to the normal components of the interface velocity in equation (C2). This leads to:

$$
\dot{m}_{S K}=-\rho_{2}\left(\boldsymbol{v}_{\mathbf{2}}^{i}-\boldsymbol{v}_{\boldsymbol{s}}\right) \cdot \widehat{\boldsymbol{n}} \text { and } \dot{m}_{L K}=-\rho_{1}\left(\boldsymbol{v}_{\mathbf{1}}^{\boldsymbol{i}}-\boldsymbol{v}_{\boldsymbol{s}}\right) \cdot \widehat{\boldsymbol{n}}
$$

- The energy balance at a point on the interface, with energy fluxes being relative to the interface, also imposes a restriction on the interfacial mass flux (denoted as $\dot{m}_{\text {energy }}$ ), and this restriction is given by:

$$
\begin{gathered}
\dot{m}_{\text {energy }}=\frac{1}{h_{s f}}\left[-\left.k_{1} \nabla T_{1}\right|^{\mathrm{i}} \cdot \widehat{\boldsymbol{n}}+-\left.k_{2} \nabla T_{2}\right|^{\mathrm{i}} \cdot \widehat{\boldsymbol{n}}\right] \\
=\frac{1}{h_{s f}}\left[-\left.k_{1} \frac{\partial T_{1}}{\partial \mathrm{n}}\right|^{\mathrm{i}}+-\left.k_{2} \frac{\partial T_{2}}{\partial \mathrm{n}}\right|^{\mathrm{i}}\right]
\end{gathered}
$$

The above equation simply states that the interfacial mass flux $\left(\mathrm{kg} /\left(\mathrm{m}^{2}-\mathrm{s}\right)\right)$ multiplied by the latent heat absorbed by the melt at the interface equals the difference between the heat-flux from the melt to the interface and the heat-flux from the interface to the solid PCM.

- Mass Balance at any point on the interface requires a single-valued interfacial massflux. That is:

$$
\dot{m}_{L K}=\dot{m}_{S K}=\dot{m}_{\text {energy }} \equiv \dot{m}
$$

Hence, substituting equations (C-6)-(C-7) in (C-8) we have,

$$
\begin{gathered}
\dot{m}_{\text {energy }}=-\rho_{1}\left(\boldsymbol{v}_{\mathbf{1}}^{i}-\boldsymbol{v}_{\boldsymbol{s}}\right) \cdot \widehat{\boldsymbol{n}}=-\rho_{2}\left(\boldsymbol{v}_{\mathbf{2}}^{\boldsymbol{i}}-\boldsymbol{v}_{\boldsymbol{s}}\right) \cdot \widehat{\boldsymbol{n}} \\
=\frac{1}{h_{s f}}\left[-\left.k_{1} \frac{\partial T_{1}}{\partial \mathrm{n}}\right|^{\mathrm{i}}+-\left.k_{2} \frac{\partial T_{2}}{\partial \mathrm{n}}\right|^{\mathrm{i}}\right]
\end{gathered}
$$

Since the solid PCM is at rest, 


$$
v_{2}^{i}=0
$$

and the normal components of the molten PCM is

$$
\boldsymbol{v}_{\mathbf{1}}^{\boldsymbol{i}}=\frac{\rho_{2}}{\rho_{1}} * \frac{\partial \emptyset / \partial t}{|\nabla \emptyset|} \widehat{\boldsymbol{n}}
$$

Substituting the values for $\boldsymbol{v}_{\mathbf{1}}^{i}, \boldsymbol{v}_{\mathbf{2}}^{i}, \frac{\partial T}{\partial n}$ and $\boldsymbol{v}_{\boldsymbol{s}} . \widehat{\boldsymbol{n}}$, in the last equality of (C-9), we get:

$$
\begin{aligned}
\rho_{2} \frac{\partial \Delta}{\partial t} * \frac{1}{\sqrt{1+\Delta_{x}^{2}}} & \\
& =\frac{1}{h s f}\left[-k_{1}\left(\left.\frac{\partial T_{1}}{\partial \mathrm{r}}\right|^{\mathrm{i}}-\left.\Delta_{x} \cdot \frac{\partial T_{1}}{\partial \mathrm{x}}\right|^{\mathrm{i}}\right)\right. \\
& \left.+k_{2}\left(\left.\frac{\partial T_{2}}{\partial \mathrm{r}}\right|^{\mathrm{i}}-\left.\Delta_{x} \cdot \frac{\partial T_{2}}{\partial \mathrm{n}}\right|^{\mathrm{i}}\right)\right] \frac{1}{\sqrt{1+\Delta_{x}^{2}}}
\end{aligned}
$$

Rearranging equation (C-12), we get:

$$
\begin{aligned}
\rho_{2} \frac{\partial \Delta}{\partial t}=\frac{1}{h s f}[ & -k_{1}\left(\left.\frac{\partial T_{1}}{\partial \mathrm{r}}\right|^{\mathrm{i}}-\left.\Delta_{x} \cdot \frac{\partial T_{1}}{\partial \mathrm{x}}\right|^{\mathrm{i}}\right) \\
& \left.+k_{2}\left(\left.\frac{\partial T_{2}}{\partial \mathrm{r}}\right|^{\mathrm{i}}-\left.\Delta_{x} \cdot \frac{\partial T_{2}}{\partial \mathrm{x}}\right|^{\mathrm{i}}\right)\right]
\end{aligned}
$$

Equation (C-13) is once again rearranged and rewritten as the interface-evolution equation (needed to track the interface between molten PCM and solid PCM) as:

where

$$
\frac{\partial \Delta}{\partial t}+\frac{\partial \Delta}{\partial x} * \bar{U}=\bar{V}
$$

and

$$
\bar{U} \equiv \frac{1}{\rho_{2} \cdot h s f}\left[-\left.k_{1} \frac{\partial T_{1}}{\partial \mathrm{x}}\right|^{\mathrm{i}}+\left.k_{2} \frac{\partial T_{2}}{\partial \mathrm{x}}\right|^{\mathrm{i}}\right]
$$

$$
\bar{V} \equiv \frac{1}{\rho_{2} \cdot h s f}\left[-\left.k_{1} \frac{\partial T_{1}}{\partial \mathrm{r}}\right|^{\mathrm{i}}+\left.k_{2} \frac{\partial T_{2}}{\partial \mathrm{r}}\right|^{\mathrm{i}}\right] \text {. }
$$

- Under negligible interfacial thermal resistance approximation and equilibrium thermodynamics assumption, the thermodynamics restriction on the interfacial temperatures requires that they equal the saturation temperature for the solid PCM (which is assumed to be at atmospheric pressure): 


$$
\left.\mathrm{T}_{1}\right|^{\mathrm{i}}=\left.\mathrm{T}_{2}\right|^{\mathrm{i}}=\mathrm{T}_{\mathrm{sat}}\left(\mathrm{p}_{\mathrm{atm}}\right)
$$

Thus, equations (C-5), (C-10), (C-11), (C-14), and (C-15) summarize the interface condition of interest to the LHTES system simulation. 


\section{Appendix D. Methods for Solving the Melt-Solid Interface Evolution Equation}

The evolution of melt-solid interface $\Delta(x, t)$ is governed by equation (C-14) of Appendix $\mathrm{C}$, that is:

$$
\frac{\partial \Delta}{\partial t}+\frac{\partial \Delta}{\partial x} * \bar{U}=\bar{V}
$$

where $\bar{U}=\frac{1}{\rho_{2} \cdot h s f}\left[-\left.k_{1} \frac{\partial T_{1}}{\partial \mathrm{x}}\right|^{\mathrm{i}}+\left.k_{2} \frac{\partial T_{2}}{\partial \mathrm{x}}\right|^{\mathrm{i}}\right]$ and $\bar{V}=\frac{1}{\rho_{2} \cdot h s f}\left[-\left.k_{1} \frac{\partial T_{1}}{\partial \mathrm{r}}\right|^{\mathrm{i}}+\left.k_{2} \frac{\partial T_{2}}{\partial \mathrm{r}}\right|^{\mathrm{i}}\right]$

The above equation is a first order hyperbolic equation with the $\frac{\partial \Delta}{\partial x} * \bar{U}$ term is often very small for many of the cases of interest here. The two ways to computationally solve the above equation are discussed below as methods I and II.

\section{Method I}

In this case, the above equation is discretized by a first order finite difference approximation for both the time and spatial derivatives of $\Delta(x, t)$. This discretization is done under the assumption that the hyperbolic (or wave) nature of the original equation can be disregarded. As shown below, this discretization results in an explicit approach for forward marching of the interface location $\Delta(x, t)$ in time. The variable $x$ is discretized as $x_{i} \equiv i * \Delta x$ (where $\left.\mathrm{i}=0,1,2, \ldots\right)$ and time $t$ is discretized as $t_{n} \equiv n \Delta t$ (where $\mathrm{n}=$ $0,1,2, \ldots)$. Hence, denoting $\Delta\left(x_{i}, t_{n}\right) \equiv \Delta\left(x_{i}, n \Delta t\right)$ and $\Delta\left(x_{i}+\Delta x, t_{n}+\Delta t\right) \equiv$ $\Delta\left(x_{i+1},(n+1) \Delta t\right)$ the evolution equation discretizes to:

$$
\begin{aligned}
\frac{\Delta\left(x_{i},(n+1) \Delta t\right)}{\Delta t} & -\Delta\left(x_{i}, n \Delta t\right) \\
= & -\bar{U}\left(x_{i}, n \Delta t\right) * \frac{\partial \Delta}{\partial x}\left(x_{i}, n \Delta t\right)+\bar{V}\left(x_{i}, n \Delta t\right)
\end{aligned}
$$

where we use the following passive estimate for the $\frac{\partial \Delta}{\partial x}$ term:

$$
\frac{\partial \Delta}{\partial x}\left(x_{i}, n \Delta t\right)=\frac{\Delta\left(x_{i+1}, n \Delta t\right)-\Delta\left(x_{i}, n \Delta t\right)}{\Delta x}
$$

The above yields the following explicit forward marching scheme: 


$$
\begin{aligned}
\Delta\left(x_{i},(n+1) \Delta t\right) & \\
& =\Delta\left(x_{i}, n \Delta t\right)+\Delta t \\
& *\left[-\bar{U}\left(x_{i}, n \Delta t\right) * \frac{\Delta\left(x_{i+1}, n \Delta t\right)-\Delta\left(x_{i}, n \Delta t\right)}{\Delta x}\right. \\
& \left.+\bar{V}\left(x_{i}, n \Delta t\right)\right]
\end{aligned}
$$

\section{Method II - Method of Characteristics}

In this method, it is recognized that the evolution equation $(\mathrm{C}-14)$ is a first order hyperbolic equation (see (Abbott 1989)) for which interfacial location values change along the characteristic curve $x=x_{c}(t)$ given by:

$$
\frac{d x_{c}(t)}{d t}=\bar{U}\left(x_{c}(t), t\right)
$$

For this method, the initial conditions for $\Delta(x, \hat{t})$ are:

$$
\Delta(x, \hat{t}=0)=\text { known }
$$

and the boundary condition is: $\quad \Delta(x=0, \hat{t})=$ known

As discussed earlier, because of the singularity at $x=0$ and $\hat{t}=0$, the known values in equation (D-5) are initially assumed (reasonable values) and then iteratively corrected from backward extrapolation of large time $\hat{t}$ solution, that are not sensitive to the initial condition in equation (D-5). The definition of the characteristic curve $x_{c}(t)$ in equation (D-4) and the interface evolution equation together imply:

$$
\begin{aligned}
\frac{d}{d t}\left[\Delta\left(x_{c}(t), t\right)\right] & =\frac{\partial \Delta}{\partial x} * \frac{d x_{c}(t)}{d x}+\frac{\partial \Delta}{\partial t} \\
& =\frac{\partial \Delta}{\partial x} * \bar{U}\left(x_{c}(t), t\right)+\frac{\partial \Delta}{\partial t}=\bar{V}\left(x_{c}(t), t\right)
\end{aligned}
$$

A simple time integration of equation (D-6) yields: 


$$
\Delta\left(x_{c}(t), t\right)=\Delta\left(x_{c}(0), 0\right)+\int_{0}^{t} \bar{V}\left(x_{c}(\bar{\tau}), \bar{\tau}\right) * d \bar{\tau}
$$

In support of the above method of characteristics a well known approach (Delhaye 1974; Narain 2004) is employed for the discretization of the time and spatial discretization of $\Delta(x, t)$. These are:

$$
\frac{\partial \Delta}{\partial t}(x, t)=\frac{\partial \Delta}{\partial t}\left(x_{i}, t_{j}\right)=\frac{1}{2}\left[\left.\frac{\partial \Delta}{\partial t}\right|_{x_{i}}+\left.\frac{\partial \Delta}{\partial t}\right|_{x_{i+1}}\right]
$$

where

$$
\begin{aligned}
& \frac{\partial \Delta}{\partial t}\left(x_{i}, t_{j}\right)= \\
& \frac{1}{2}\left[\left(\frac{\Delta\left(x_{i}, t_{j+1}\right)-\Delta\left(x_{i}, t_{j}\right)}{t_{j+1}-t_{j}}\right)+\left(\frac{\Delta\left(x_{i+1}, t_{j+1}\right)-\Delta\left(x_{i+1}, t_{j}\right)}{t_{j+1}-t_{j}}\right)\right] \\
& \text { and } \quad \frac{\partial \Delta}{\partial x}(x, t)=\frac{\partial \Delta}{\partial x}\left(x_{i}, t_{j}\right)=\frac{1}{2}\left[\left.\frac{\partial \Delta}{\partial x}\right|_{t_{j}}+\left.\frac{\partial \Delta}{\partial x}\right|_{t_{j+1}}\right] \\
& \quad=\frac{1}{2}\left[\left(\frac{\Delta\left(x_{i+1}, t_{j}\right)-\Delta\left(x_{i}, t_{j}\right)}{x_{i+1}-x_{i}}\right)+\left(\frac{\Delta\left(x_{i+1}, t_{j+1}\right)-\Delta\left(x_{i}, t_{j+1}\right)}{x_{i+1}-x_{i}}\right)\right]
\end{aligned}
$$

Employing the notations $\bar{U}(x, t)=\bar{U}\left(x_{i}, t_{j}\right) \equiv \bar{U}_{i, j}$ and $\bar{V}(x, t)=\bar{V}\left(x_{i}, t_{j}\right) \equiv \bar{V}_{i, j}$ and substituting the above expressions in the evolution equation (D-0), one obtains:

$$
\begin{aligned}
& \frac{1}{2}\left[\frac{\Delta_{i, j+1}-\Delta_{i, j}}{\Delta t}+\frac{\Delta_{i+1, j+1}-\Delta_{i+1, j}}{\Delta t}\right]+\bar{U}_{i, j} * \frac{1}{2}\left[\frac{\Delta_{i+1, j}-\Delta_{i, j}}{\Delta x}+\right. \\
& \Delta i+1, j+1-\Delta i, j+1 \Delta x=V i, j
\end{aligned}
$$

Multiplying both sides by $2 \Delta t$ we have:

$$
\begin{gathered}
{\left[\Delta_{i, j+1}-\Delta_{i, j}+\Delta_{i+1, j+1}-\Delta_{i+1, j}\right]+\bar{U}_{i, j}} \\
* \frac{1}{2}\left[\Delta_{i+1, j}-\Delta_{i, j}+\Delta_{i+1, j+1}-\Delta_{i, j+1}\right] \\
=\bar{V}_{i, j} * 2 \Delta t
\end{gathered}
$$

Hence, 


$$
\begin{aligned}
& \Delta_{i, j+1}\left[1+C r_{i, j}\right]+\Delta_{i, j}\left[-1-C r_{i, j}\right]+\Delta_{i+1, j+1}\left[1-C r_{i, j}\right]+ \\
& \Delta_{i+1, j}\left[-1+C r_{i, j}\right]=\bar{V}_{i, j} * 2 \Delta t
\end{aligned}
$$

where $C r_{i, j}=\bar{U}_{i, j} \frac{\Delta t}{\Delta x}$ is the well known Courant number (Abbott 1989). The solution scheme is sequential in time and space and marching in time is properly done. It is given by:

$$
\begin{gathered}
\Delta_{i, j+1} \\
=\frac{1}{\left[1+C r_{i, j}\right]}\left[\bar{V}_{i, j} * 2 \Delta t+\Delta_{i, j}\left[1+C r_{i, j}\right]-\Delta_{i+1, j+1}\left[1-C r_{i, j}\right]\right. \\
\left.-\Delta_{i+1, j}\left[-1+C r_{i, j}\right]\right]
\end{gathered}
$$

For both convergence as well as phase stability (see (Abbott 1989)), in order to capture interface waves (if any), $\Delta x$ is chosen such that $C r_{i, j} \approx 1$. Therefore, for $C r_{i, j} \approx 1$, we have:

$$
\begin{gathered}
\Delta_{i, j+1} \cong \frac{1}{2}\left[\bar{V}_{i, j} * 2 \Delta t+2 \Delta_{i, j}\right] \\
=\Delta_{i, j}+\bar{V}_{i, j} * \Delta t
\end{gathered}
$$

The expressions in (D-14) and (D-15) are used to obtain evolution of the interface $\Delta(x, t)$ with time and these expressions captures the essential analytical result in equation (D-7). 